$\underline{\text { Research Article }}$

\title{
Secular changes in child marriage and secondary school completion among rural adolescent girls in India
}

\author{
Tara S Beattie ${ }^{1}$, Prakash Javalkar ${ }^{2}$, Mitzy Gafos ${ }^{1}$, Lori Heise ${ }^{3}$, Stephen Moses ${ }^{4}$, Ravi Prakash ${ }^{2}$ \\ 1 London School of Hygiene \& Tropical Medicine, Department of Global Health and Development, London, UK, 2 Karnataka Health Promotion Trust, \\ Bangalore, India, ${ }^{3}$ Johns Hopkins Bloomberg School of Public Health and JHU School of Nursing, Department of Population, Family and Reproductive \\ Health, 615 N. Wolfe Street, Baltimore, Maryland, USA, ${ }^{4}$ University of Manitoba, Department of Community Medicine, Manitoba, Canada \\ Keywords: global health \\ https://doi.org/10.29392/joghr.3.e2019041
}

\section{Journal of Global Health Reports}

Vol. 3, 2019

\section{Background}

Child marriage ( $<18$ years) and school drop-out disproportionately affect girls living in impoverished households in rural areas, with long-term economic and health consequences. Improving retention in education, and delaying age at marriage and first pregnancy have received substantial attention at the national and global level, in line with the Millennium Development Goals and the Sustainable Development Goals (SDGs) (2015-2030).

\section{Methods}

We examined changes over time in economic, education and child marriage indicators among adolescents from rural households in (i) Northern Karnataka (the most deprived region of Karnataka), (ii) Karnataka state, and (iii) all India, using individualized data from four pre-existing, nationally-representative datasets (District Level Household and Facility Surveys (DLHS 2-4) (2002/4-2012/3) and the National Family Health Survey (NFHS-4) (2015-16)).

\section{Results}

At the national and state level, we found large improvements in secondary educational attainment among girls and boys living in rural settings (proportion of adolescents completing age-appropriate secondary school education (all India): girls 12.4\% 2002/3 vs. $31.6 \% 2015 / 6$; boys $18.9 \% 2002 / 4$ vs. $36.8 \% 2015 / 6$ ). We also observed large reductions in child marriage and early child-bearing rates (proportion of married women aged 18-24 years married < 18 years: $62.4 \% 2002 / 4$ vs. $23.8 \% 2015 / 6$; proportion of married girls aged $<19$ years who are pregnant or have children: $62.4 \%$ 2002/4 vs. $21.9 \% 2015 / 6$ ). In addition, we found evidence of "clustered deprivations", whereby girls in rural areas from the poorest families and lowest castes continue to experience multiple forms of disadvantage, with child marriage significantly associated with scheduled caste / scheduled tribe (SC/ST) caste (odds ratio $(\mathrm{OR})=1.25,95 \%$ confidence interval $(\mathrm{CI})=1.18-1.32)$, poorest quintile $(\mathrm{OR}=2.38,95 \% \mathrm{CI}=2.21-2.55)$ and illiteracy $(\mathrm{OR}=2.09,95 \% \mathrm{CI}=1.95-2.23)$; and not completing secondary education significantly associated with SC/ST caste (OR=1.52, 95\% $\mathrm{CI}=1.45-1.59)$, poorest quintile $(\mathrm{OR}=4.17,95 \% \mathrm{CI}=3.90-4.46)$, and child marriage $(\mathrm{OR}=2.05,95 \% \mathrm{CI}=1.85-2.26)$.

\section{Conclusions}

The results show substantial improvements in economic, educational and child marriage indicators at the state and national level over the past 14 years. The government has implemented multiple programmes and policies to address child marriage and school drop-out, and these trends suggest such efforts may be having a positive impact. If India is to achieve the SDGs, designing targeted interventions to reach those who continue to be left furthest behind is going to be key.

Child marriage, defined as marriage under the age of 18 years, is associated with a range of adverse economic and health outcomes, including inter-generational poverty, early and inadequately spaced pregnancies, intimate partner violence, poor mental health, poor utilization of maternal health services, maternal and child mortality, and 
child malnutrition. ${ }^{1-6}$ The practice disproportionately affects girls from impoverished families in low and middleincome countries (LMIC), particularly those living in rural and slum areas with low access to health care and education. 1,6 Estimates of global trends in child marriage suggest a slow reduction in incidence, with rates usually far higher in rural compared with urban settings. ${ }^{7}$ In 2010 , it was estimated that one- quarter (26\%) of women aged $20-24$ years were married as children globally, with one-third of all child marriages taking place in India. ${ }^{8}$ In Asian settings, child marriage is driven by structural and social factors, including poverty and underlying gender norms around sexual purity and family honour, which come to the fore once a girl reaches menarche. ${ }^{9}$ Young brides often marry men who are much older and move away from their natal home and social support to live with their spouse. ${ }^{3,6}$ Married girls assume new roles of wife, mother, and homemaker, with marriage typically marking the end of a girl's education. ${ }^{10,11}$

The past 15 years have seen rapid progress towards universal primary education, with narrowing gender gaps and increased opportunities for disadvantaged groups. ${ }^{12}$ Globally, the adjusted net enrolment rate in primary education among primary-aged children grew from $83 \%$ in 2000 to $90 \%$ in $2011 .{ }^{13}$ However, 57 million children still need to be reached to achieve universal primary school access. ${ }^{13}$ Nonetheless, following improvements in primary education access, the policy focus is shifting to improving parity in access and completion of quality secondary school education. ${ }^{12}$ Access to education is one of the strongest determinants of adolescent health. ${ }^{14}$ Quality secondary school education is causally associated with a range of positive economic and health outcomes, including improved livelihood and economic options, improved nutrition, improved ability to control fertility, reduced HIV and STI infection, reduced intimate partner violence (IPV), and improved maternal and child survival rates. ${ }^{11,14-17}$ Indeed, improvements in education among women of reproductive age was associated with a halving (51.2\%) of global child mortality rates between 1970 and 2009. ${ }^{16}$

Drop-out from school is usually the result of a process, rather than a single event, with more than one proximate cause. ${ }^{18}$ Similar to child marriage, rural location, poverty and gender norms are key determinants globally of school drop-out, with girls disproportionately affected. ${ }^{18,19}$ Poverty and the critical need for child labour can lead to frequent absenteeism and subsequent withdrawal from school, especially if children fall behind academically. ${ }^{18,20}$ Among families with limited resources, investment in boys' education is often prioritised, fuelled by the belief that investment in girls is lost once they marry and move to their spouse's home. ${ }^{20,21}$ In India, secondary school drop-out is also linked to menarche, with concerns around sexual purity and family honour leading to restriction of girls' mobility and withdrawal from school.9,20 Girls from scheduled castes and scheduled tribes (SC/ST) - the lowest castes in the Hindu caste system - living in rural areas, can be particularly at risk. These families are usually the most impoverished and marginalised. Dwellings are often located outside main villages, for example in the agricultural fields, presenting additional economic and distance/ time-related barriers to accessing education.
Over the past 15 years, India has experienced substantial improvements in economic growth, with rapid development and expansion of major cities. Internet and mobile phone availability in rural and urban settings has increased exponentially. Additionally, state and national governments have introduced a range of legislative and policy changes, as well as programming designed to address child marriage and improve educational outcomes. This includes the Prohibition of Child Marriage Act (2006), ${ }^{22}$ which made marrying a female minor, conducting a marriage that involves a minor and permitting your child to be married as a minor, illegal and punishable by law (jail or fine). In addition, The Right of Children (aged 6 to 14 years) to Free and Compulsory Education Act (2009), ${ }^{23}$ effectively removed primary and lower secondary school fees. There have also been national campaigns promoting gender equality and girls' education, the most notable of which are the nationwide "Beto Bachao Beti Padhao (save your daughter, educate your daughter)" programme launched in 2015. Economic schemes designed to support students in government and government-aided schools to remain in school have also been implemented, such as free midday meals, free bicycles, free school uniforms, and scholarships for children from SC/ ST families. ${ }^{24}$

In 2013-2017, we conducted a cluster-randomized controlled trial (RCT) in two rural districts in north Karnataka, south India, to evaluate Samata, a multi-level structural and social norms intervention designed to reduce child marriage and secondary school drop-out among SC/ST adolescent girls. ${ }^{23,25}$ At trial end, we found no impact on child marriage or girl retention in secondary school education, and child marriage and school drop-out rates at trial end were far lower than we anticipated (based on district level data available at the start of the trial) in both the intervention and comparison communities. ${ }^{26}$ We hypothesised that this may have been due to changes in secular trends regarding economic indicators, school retention and child marriage but were not able to find studies which used representative data from after 2005, at the district, state or national level, to support this. We undertook the current analyses to understand the broader context within which this trial took place. Specifically we wanted to examine (i) levels and trends in economic, marriage, sexual and reproductive health and education indicators among rural adolescent girls over the past 15 years; (ii) levels and trends in education indicators among rural adolescent boys; (iii) if trends observed in northern Karnataka were also seen across the state, and India as a whole; and (iv) key social factors associated with education and marriage outcomes among rural adolescent girls.

\section{METHODS}

We undertook secondary analysis of pre-existing, nationally-representative datasets from three rounds of India's District Level Household and Facility Surveys (DLHS) conducted from 2002 to 2013 (DLHS-2 in 2002-04, DLHS-3 in 2007-08, DLHS-4 in 2012-13), and the most recent round of the National Family Health Survey (NFHS) conducted in 2015-16 (NFHS-4), to estimate trends in economic, marriage, sexual and reproductive health indicators, as well as 
education-related indicators among rural adolescents. We chose these surveys for the analysis as they are conducted periodically and provide estimates on reproductive and child health programme indicators at national and sub-national levels across India. Both the DLHS and NFHS-4 surveys employ a similar systematic, multi-stage stratified sampling scheme, whereby villages in rural areas and Census Enumeration Blocks in urban areas were the primary sampling units (PSU) and were selected using a probability proportional to size (PPS) methodology. The required number of households within each PSU were then selected randomly using a systematic random sampling methodology. The use of such a multistage sampling approach helps ensure the representativeness of the sample and avoids selection bias. Survey weights were used to adjust for nonresponse and the multistage stratified sampling design, in order to make the estimates representative at the state and district levels. The DLHS and NFHS-4 surveys were implemented by the Ministry of Health and Family Welfare (MoHFW) through the International Institute for Population Sciences (IIPS) as a lead agency along with other national and international development partners.

The DLHS (2002 to 2013) provides estimates on household socio-demographic indicators, along with maternal and child health programme indicators, across every district in India. However, the DLHS-IV survey (2012-13) did not collect data from eight of the poorest performing states in India as data from these states were collected using a different survey. The NFHS surveys were meant to provide state and national level estimates only, but, the latest survey round (NFHS-IV, 2015-16), also provided estimates at the district level, and thereby replaced the DLHS and thus were included in the current analysis. Additional detail on the purpose, survey design, methodology, and results are available elsewhere. ${ }^{27}$

For both surveys (DLHS and NFHS-IV), interviews were conducted with ever-married women (aged 15-49 years) for the "women's questionnaire" and with any adult family member (aged 18+ years) for the "household questionnaire". We used information from the women's questionnaire to obtain child marriage, co-habitation, child-bearing and mothers' literacy rates. Data from the household questionnaire contained relevant information on socio-economic characteristics of the household and educational attainment details of household members (including adolescents).

All analyses were carried out using STATA version 14.0 (Stata Corporation, College Station, TX, USA). To examine changes in the levels and trends in economic, marriage, sexual and reproductive health and education indicators among rural adolescents, we used individual and household level data from the 4 surveys and conducted weighted analyses (using the state and district level weights available with the dataset) for nine rural districts in northern Karnataka (as these are the most disadvantaged districts in Karnataka state ${ }^{28}$ ), all districts in Karnataka, and all districts across India, and stratified analyses based on gender and caste. Results are presented in the form of weighted crude percentages.

To examine current associations between education / marriage outcomes among adolescent rural girls and key so- cial factors (caste, wealth, literacy, marriage) we conducted cross-sectional analyses at the state and national level using data from the most recent survey round (NFHS-4) using logistic regression. Results are presented in the form of crude odds-ratio (OR) and 95\% confidence intervals. We did not adjust these analyses for confounding factors as the purpose of this analysis was to understand which social factors were associated with school drop-out and child marriage.

\section{RESULTS}

\section{SECULAR CHANGES IN ECONOMIC INDICATORS}

We examined 6 economic indicators among SC/ST and nonSC/ST rural households over a 14-year period (2002-2016). In northern Karnataka, we found a dramatic step-wise increase in the proportion of rural households with electricity (79.8\% vs. 97.0\%), phone access (landline or mobile) $(6.4 \%$ vs. $86.9 \%$ ), and any of 3 assets (TV, fan, refrigerator) (33.2\% vs. $81.5 \%$ ); this was true both for non-SC/ST and for SC/ ST households. These trends were also seen across Karnataka state and India (Table 1). Likewise, although not always directly linear, there was a dramatic increase between $2002 / 3$ and 2015/6 in the proportion of rural households in northern Karnataka who use clean fuel for cooking (8.9\% vs. $17.6 \%)$, who have use of a toilet facility (8.6\% vs. $21.6 \%)$, and who live in a house fully or partially constructed with solid building materials (pucca or semi-pucca) (76.6\% vs. 94.0\%), with these increases seen for both non-SC/ST and SC/ST households. However, a higher proportion of non-SC/ ST households compared with SC/ST households reported attaining these 6 different economic indicators, reflecting caste disparities in economic attainment. Similar increases and caste disparities were also seen among rural households across Karnataka state and India (Table 2 and Table 3 ).

Secular changes in child marriage, cohabitation and child-bearing

There has been a substantial step-wise reduction in the proportion of women aged 18-24 years who were married by 18 years ( $77.4 \%$ vs. $26.7 \%)$, and who were married and cohabiting by 18 years ( $77.4 \%$ vs. $25.6 \%$ ), as well as substantial reductions in the proportion of married adolescent girls (<19 years) who were pregnant or had children $(59.9 \%$ vs. 5.0\%) in rural northern Karnataka between $2002 / 3$ and 2015/6 (Table 1). Literacy rates among mothers (15-49 years) significantly improved during this 14-year period (25.0\% vs. $44.0 \%$ ). These improvements were also seen among non-SC/ST and SC/ST females across Karnataka state and India (Tables $\underline{2}$ and $\underline{3}$ ). Although the gap is diminishing, caste disparities remain, with rural SC/ST girls having higher rates of child marriage, cohabitation and childbearing, and lower literacy rates, compared with rural non-SC/ST girls. At each of the four-time points, child marriage rates were far higher and maternal literacy rates were far lower in northern Karnataka, compared with Karnataka state and all India (Table 1).

\section{SECULAR CHANGES IN EDUCATIONAL ATTAINMENT}

There have been dramatic step-wise improvements in educational attainment among boys and girls living in rural ar- 


\section{Table 1. Profile of all rural girls}

\begin{tabular}{|c|c|c|c|c|c|c|c|c|c|c|c|c|}
\hline \multirow{4}{*}{ Indicators } & \multicolumn{4}{|c|}{$\begin{array}{l}\text { Northern Karnataka Districts } \\
\text { (9 districts)* }\end{array}$} & \multicolumn{4}{|c|}{ Karnataka } & \multicolumn{4}{|c|}{ India } \\
\hline & $\underset{\|}{\text { DLHS }}$ & $\begin{array}{c}\text { DLHS } \\
\text { III }\end{array}$ & $\begin{array}{l}\text { DLHS } \\
\text { IV } \dagger\end{array}$ & $\begin{array}{l}\text { NFHS } \\
\text { IV }\end{array}$ & DLHS II & DLHSIII & $\begin{array}{l}\text { DLHS } \\
\text { IV } \dagger\end{array}$ & $\begin{array}{l}\text { NFHS } \\
\text { IV }\end{array}$ & DLHS II & DLHS III & $\begin{array}{l}\text { DLHS } \\
\text { IV } \dagger\end{array}$ & NFHS IV \\
\hline & $2002 / 3$ & $2007 / 8$ & $2012 / 3$ & $2015 / 6$ & $2002 / 3$ & $2007 / 8$ & $2012 / 3$ & $2015 / 6$ & $2002 / 3$ & $2007 / 8$ & $2012 / 3$ & $2015 / 6$ \\
\hline & $\%(N)$ & $\%(N)$ & $\%(N)$ & $\%(N)$ & $\%(N)$ & $\%(N)$ & $\%(N)$ & $\%(N)$ & $\%(N)$ & $\%(N)$ & $\%(N)$ & $\%(N)$ \\
\hline \multicolumn{13}{|l|}{ Economic: } \\
\hline $\mathrm{N}$ & 5747 & 6469 & 8939 & 4419 & 18733 & 20904 & 26540 & 14490 & 415135 & 548694 & 217904 & 408296 \\
\hline Proportion of $\mathrm{HH}$ with electricity & $\begin{array}{c}79.8 \\
(4601)\end{array}$ & $\begin{array}{c}87.2 \\
(5617)\end{array}$ & $\begin{array}{c}93.6 \\
(8353)\end{array}$ & $\begin{array}{c}97.0 \\
(4292)\end{array}$ & $\begin{array}{c}83.1 \\
(15466)\end{array}$ & $\begin{array}{c}86.2 \\
(18022)\end{array}$ & $\begin{array}{c}94.7 \\
(25118)\end{array}$ & $\begin{array}{c}96.9 \\
(14012)\end{array}$ & $\begin{array}{c}63.5 \\
(233098)\end{array}$ & $\begin{array}{c}60.1 \\
(323517)\end{array}$ & $\begin{array}{c}93.7 \\
(203725)\end{array}$ & $\begin{array}{c}83.0 \\
(342631)\end{array}$ \\
\hline Proportion of $\mathrm{HH}$ having any phone & $\begin{array}{c}6.4 \\
(392)\end{array}$ & $\begin{array}{c}27.9 \\
(1795)\end{array}$ & $\begin{array}{c}80.5 \\
(7197)\end{array}$ & $\begin{array}{c}86.9 \\
(3801)\end{array}$ & $\begin{array}{c}10.0 \\
(2090)\end{array}$ & $\begin{array}{c}34.2 \\
(7128)\end{array}$ & $\begin{array}{c}83.3 \\
(22125)\end{array}$ & $\begin{array}{c}88.5 \\
(12762)\end{array}$ & $\begin{array}{c}7.6 \\
(36821)\end{array}$ & $\begin{array}{c}29.0 \\
(157838)\end{array}$ & $\begin{array}{c}82.1 \\
(179043)\end{array}$ & $\begin{array}{c}87.4 \\
(354978)\end{array}$ \\
\hline Proportion of $\mathrm{HH}$ with any of 3 assets (TV, Fan, Refrigerator) & $\begin{array}{c}33.2 \\
(1978)\end{array}$ & $\begin{array}{c}43.5 \\
(2728)\end{array}$ & $\begin{array}{c}56.4 \\
(5061)\end{array}$ & $\begin{array}{c}81.5 \\
(3582)\end{array}$ & $\begin{array}{c}39.0 \\
(7421)\end{array}$ & $\begin{array}{c}48.3 \\
(10085)\end{array}$ & $\begin{array}{c}68.3 \\
(18135)\end{array}$ & $\begin{array}{c}83.3 \\
(12012)\end{array}$ & $\begin{array}{c}48.3 \\
(177761)\end{array}$ & $\begin{array}{c}43.8 \\
(237650)\end{array}$ & $\begin{array}{c}73.1 \\
(159436)\end{array}$ & $\begin{array}{c}72.9 \\
(286027)\end{array}$ \\
\hline Proportion of $\mathrm{HH}$ using clean fuel for cooking & $\begin{array}{l}8.9 \\
(539)\end{array}$ & $\begin{array}{c}3.5 \\
(204)\end{array}$ & $\begin{array}{c}7.6 \\
(683)\end{array}$ & $\begin{array}{l}17.6 \\
(711)\end{array}$ & $\begin{array}{c}12.3 \\
(2510)\end{array}$ & $\begin{array}{c}7.9 \\
(1634)\end{array}$ & $\begin{array}{c}19.6 \\
(5210)\end{array}$ & $\begin{array}{c}32.0 \\
(4394)\end{array}$ & $\begin{array}{c}13.8 \\
(56483)\end{array}$ & $\begin{array}{c}8.8 \\
(47258)\end{array}$ & $\begin{array}{c}28.4 \\
(61886)\end{array}$ & $\begin{array}{c}24.1 \\
(86873)\end{array}$ \\
\hline Proportion of $\mathrm{HH}$ uses a toilet facility & $\begin{array}{l}8.6 \\
(524)\end{array}$ & $\begin{array}{c}6.0 \\
(342)\end{array}$ & $\begin{array}{c}9.0 \\
(810)\end{array}$ & $\begin{array}{l}21.6 \\
(962)\end{array}$ & $\begin{array}{c}19.2 \\
(4087)\end{array}$ & $\begin{array}{c}23.0 \\
(4771)\end{array}$ & $\begin{array}{c}35.2 \\
(9391)\end{array}$ & $\begin{array}{c}47.1 \\
(6973)\end{array}$ & $\begin{array}{c}19.2 \\
(126244)\end{array}$ & $\begin{array}{c}33.3 \\
(178274)\end{array}$ & $\begin{array}{c}64.0 \\
(140973)\end{array}$ & $\begin{array}{c}44.8 \\
(201030)\end{array}$ \\
\hline Proportion of HH has Pakka/Semi Pakka house & $\begin{array}{c}76.6 \\
(4454)\end{array}$ & $\begin{array}{c}63.7 \\
(4066)\end{array}$ & $\begin{array}{c}81.6 \\
(7300)\end{array}$ & $\begin{array}{c}94.0 \\
(4117)\end{array}$ & $\begin{array}{c}84.1 \\
(16036)\end{array}$ & $\begin{array}{c}72.2 \\
(15080)\end{array}$ & $\begin{array}{c}84.1 \\
(22371)\end{array}$ & $\begin{array}{c}95.6 \\
(13725)\end{array}$ & $\begin{array}{c}60.1 \\
(227606)\end{array}$ & $\begin{array}{c}54.0 \\
(294471)\end{array}$ & $\begin{array}{c}74.4 \\
(161210)\end{array}$ & $\begin{array}{c}91.5 \\
(354719)\end{array}$ \\
\hline \multicolumn{13}{|l|}{ Marriage: } \\
\hline $\mathrm{N}$ & 1641 & 1865 & 2283 & 1298 & 4388 & 4521 & 4839 & 3596 & 92517 & 111239 & 29931 & 118688 \\
\hline Proportion of women aged $18-24$ who were married below 18 years & $\begin{array}{c}77.4 \\
(1264)\end{array}$ & $\begin{array}{c}74.9 \\
(1401)\end{array}$ & $\begin{array}{c}52.0 \\
(1192)\end{array}$ & $\begin{array}{l}26.7 \\
(345)\end{array}$ & $\begin{array}{c}64.0 \\
(2810)\end{array}$ & $\begin{array}{c}58.5 \\
(2650)\end{array}$ & $\begin{array}{c}42.2 \\
(2043)\end{array}$ & $\begin{array}{l}21.8 \\
(758)\end{array}$ & $\begin{array}{c}62.4 \\
(53729)\end{array}$ & $\begin{array}{c}51.7 \\
(58114)\end{array}$ & $\begin{array}{c}34.0 \\
(10100)\end{array}$ & $\begin{array}{c}23.8 \\
(25415)\end{array}$ \\
\hline \multicolumn{13}{|l|}{ SRH: } \\
\hline $\mathrm{N}$ & 1641 & 1865 & 2283 & 1298 & 4388 & 4521 & 4839 & 3596 & 92517 & 111239 & 29931 & 118688 \\
\hline $\begin{array}{l}\text { Proportion of women aged } 18-24 \text { who were married }<18 \text { years and } \\
\text { co-habiting }<18 \text { years }\end{array}$ & $\begin{array}{c}77.4 \\
(1264)\end{array}$ & $\begin{array}{c}72.9 \\
(1366)\end{array}$ & $\begin{array}{c}48.3 \\
(1109)\end{array}$ & $\begin{array}{l}25.6 \\
(329)\end{array}$ & $\begin{array}{c}64.0 \\
(2810)\end{array}$ & $\begin{array}{c}57.5 \\
(2605)\end{array}$ & $\begin{array}{c}39.2 \\
(1903)\end{array}$ & $\begin{array}{l}20.8 \\
(724)\end{array}$ & $\begin{array}{c}62.4 \\
(53729)\end{array}$ & $\begin{array}{c}46.5 \\
(52169)\end{array}$ & $\begin{array}{c}32.2 \\
(9562)\end{array}$ & $\begin{array}{c}21.9 \\
(23256)\end{array}$ \\
\hline $\mathrm{N}$ & 705 & 632 & 489 & 659 & 1295 & 1050 & 762 & 1894 & 22026 & 22818 & 3346 & 72534 \\
\hline $\begin{array}{l}\text { Proportion of married girls aged }<19 \text { years pregnant or have } \\
\text { children }\end{array}$ & $\begin{array}{l}59.9 \\
(419)\end{array}$ & $\begin{array}{l}56.0 \\
(360)\end{array}$ & $\begin{array}{l}53.8 \\
(265)\end{array}$ & $\begin{array}{l}5.0 \\
(34)\end{array}$ & $\begin{array}{l}56.5 \\
(763)\end{array}$ & $\begin{array}{l}53.9 \\
(566)\end{array}$ & $\begin{array}{l}52.6 \\
(404)\end{array}$ & $5.8(96)$ & $\begin{array}{c}52.8 \\
(11471)\end{array}$ & $\begin{array}{c}47.6 \\
(10905)\end{array}$ & $\begin{array}{c}49.6 \\
(1680)\end{array}$ & $\begin{array}{c}5.3 \\
(3404)\end{array}$ \\
\hline $\mathrm{N}$ & 4321 & 5943 & 8165 & 3606 & 13510 & 18264 & 22650 & 11285 & 305792 & 443664 & 162793 & 328344 \\
\hline Proportion of Mothers who are literate & $\begin{array}{c}25.0 \\
(1125)\end{array}$ & $\begin{array}{c}33.3 \\
(1938)\end{array}$ & $\begin{array}{c}38.2 \\
(3137)\end{array}$ & $\begin{array}{c}44.0 \\
(1576)\end{array}$ & $\begin{array}{c}40.4 \\
(5683)\end{array}$ & $\begin{array}{c}49.4 \\
(9003)\end{array}$ & $\begin{array}{c}54.9 \\
(12468)\end{array}$ & $\begin{array}{c}60.4 \\
(6763)\end{array}$ & $\begin{array}{c}39.3 \\
(127072)\end{array}$ & $\begin{array}{c}46.3 \\
(202869)\end{array}$ & $\begin{array}{c}63.2 \\
(103528)\end{array}$ & $\begin{array}{c}56.6 \\
(187688)\end{array}$ \\
\hline \multicolumn{13}{|l|}{ Education: } \\
\hline $\mathrm{N}$ & 1279 & 1377 & 1573 & 676 & 3408 & 3552 & 3755 & 1799 & 80595 & 106097 & 26858 & 62928 \\
\hline Proportion of girls aged 12-14 entered into secondary school & $\begin{array}{l}12.8 \\
(162)\end{array}$ & $\begin{array}{l}18.9 \\
(256)\end{array}$ & $\begin{array}{l}22.6 \\
(360)\end{array}$ & $\begin{array}{l}26.9 \\
(175)\end{array}$ & $\begin{array}{l}23.9 \\
(798)\end{array}$ & $\begin{array}{l}26.0 \\
(922)\end{array}$ & $\begin{array}{c}36.0 \\
(1361)\end{array}$ & $\begin{array}{l}33.5 \\
(606)\end{array}$ & $\begin{array}{c}24.9 \\
(13569)\end{array}$ & $\begin{array}{c}19.3 \\
(20250)\end{array}$ & $\begin{array}{c}39.3 \\
(10476)\end{array}$ & $\begin{array}{c}28.1 \\
(16903)\end{array}$ \\
\hline
\end{tabular}




\begin{tabular}{|c|c|c|c|c|c|c|c|c|c|c|c|c|}
\hline $\mathrm{N}$ & 1269 & 1311 & 1449 & 477 & 3441 & 3371 & 3512 & 1462 & 73205 & 85319 & 25834 & 57075 \\
\hline Proportion of girls aged 15-17 completed secondary education & $\begin{array}{c}15.3 \\
(209)\end{array}$ & $\begin{array}{l}23.8 \\
(300)\end{array}$ & $\begin{array}{l}33.0 \\
(483)\end{array}$ & $\begin{array}{l}41.5 \\
(197)\end{array}$ & $\begin{array}{l}23.4 \\
(848)\end{array}$ & $\begin{array}{c}34.4 \\
(1159)\end{array}$ & $\begin{array}{c}49.2 \\
(1729)\end{array}$ & $\begin{array}{l}55.8 \\
(815)\end{array}$ & $\begin{array}{c}21.9 \\
(11974)\end{array}$ & $\begin{array}{c}21.6 \\
(18249)\end{array}$ & $\begin{array}{c}49.6 \\
(12781)\end{array}$ & $\begin{array}{c}36.5 \\
(19331)\end{array}$ \\
\hline $\mathrm{N}$ & 1461 & 1604 & 2040 & 627 & 4524 & 4354 & 4496 & 1740 & 87567 & 101247 & 29786 & 59931 \\
\hline $\begin{array}{l}\text { Proportion of girls aged } 18-20 \text { completed higher-secondary } \\
\text { education }\end{array}$ & $\begin{array}{c}7.6 \\
(116)\end{array}$ & $\begin{array}{c}9.8 \\
(150)\end{array}$ & $\begin{array}{l}17.6 \\
(365)\end{array}$ & $\begin{array}{l}26.6 \\
(168)\end{array}$ & $\begin{array}{l}10.8 \\
(538)\end{array}$ & $\begin{array}{l}17.2 \\
(749)\end{array}$ & $\begin{array}{c}29.7 \\
(1353)\end{array}$ & $\begin{array}{r}38.4 \\
(673)\end{array}$ & $\begin{array}{c}12.4 \\
(8993)\end{array}$ & $\begin{array}{c}14.0 \\
(14149)\end{array}$ & $\begin{array}{c}37.5 \\
(11213)\end{array}$ & $\begin{array}{c}31.6 \\
(17822)\end{array}$ \\
\hline
\end{tabular}

DLHS - District Level Household and Facility Surveys, NFHS - National Family Health Survey, HH - Household, SRH - Sexual and Reproductive Health "9 districts include - Bidar, Gulbarga, Bijapur, Yadgir, Bagalkot, Raichur, Gadag, Koppal and Bellary.

$\uparrow$ Data not collected from 8 poorly performing States in India (as data from these States were collected in a different survey). 
Table 2. Profile of rural non-Scheduled Caste/Scheduled Tribe girls

\begin{tabular}{|c|c|c|c|c|c|c|c|c|c|c|c|c|}
\hline \multirow{4}{*}{ Indicators } & \multicolumn{4}{|c|}{$\begin{array}{l}\text { Northern Karnataka Districts } \\
\text { (9 districts) }\end{array}$} & \multicolumn{4}{|c|}{ Karnataka } & \multicolumn{4}{|c|}{ India } \\
\hline & $\begin{array}{l}\text { DLHS } \\
\|\end{array}$ & $\begin{array}{c}\text { DLHS } \\
\text { III }\end{array}$ & $\begin{array}{l}\text { DLHS } \\
\text { IV } †\end{array}$ & $\begin{array}{l}\text { NFHS } \\
\text { IV }\end{array}$ & DLHSII & DLHSIII & $\begin{array}{l}\text { DLHS } \\
\text { IV } \dagger\end{array}$ & $\begin{array}{l}\text { NFHS } \\
\text { IV }\end{array}$ & DLHS II & DLHS III & $\begin{array}{l}\text { DLHS } \\
\text { IV } \dagger\end{array}$ & NFHSIV \\
\hline & $2002 / 3$ & $2007 / 8$ & $2012 / 3$ & $2015 / 6$ & $2002 / 3$ & $2007 / 8$ & $2012 / 3$ & $2015 / 6$ & $2002 / 3$ & $2007 / 8$ & $2012 / 3$ & $2015 / 6$ \\
\hline & $\%(N)$ & $\%(N)$ & $\%(\mathrm{~N})$ & $\%(N)$ & $\%(N)$ & $\%(\mathrm{~N})$ & $\%(N)$ & $\%(N)$ & $\%(N)$ & $\%(\mathrm{~N})$ & $\%(\mathrm{~N})$ & $\%(N)$ \\
\hline \multicolumn{13}{|l|}{ Economic: } \\
\hline $\mathrm{N}$ & 3956 & 4198 & 5464 & 2497 & 13364 & 14678 & 17212 & 9108 & 255655 & 321487 & 110035 & 233383 \\
\hline Proportion of $\mathrm{HH}$ with electricity & $\begin{array}{c}82.0 \\
(3259)\end{array}$ & $\begin{array}{c}88.0 \\
(3677)\end{array}$ & $\begin{array}{c}94.0 \\
(5131)\end{array}$ & $\begin{array}{c}97.2 \\
(2429)\end{array}$ & $\begin{array}{c}86.1 \\
(11431)\end{array}$ & $\begin{array}{c}88.1 \\
(12936)\end{array}$ & $\begin{array}{c}95.4 \\
(16419)\end{array}$ & $\begin{array}{c}97.6 \\
(8880)\end{array}$ & $\begin{array}{c}67.0 \\
(151746)\end{array}$ & $\begin{array}{c}62.8 \\
(198366)\end{array}$ & $\begin{array}{c}96.3 \\
(106033)\end{array}$ & $\begin{array}{c}84.5 \\
(198400)\end{array}$ \\
\hline Proportion of $\mathrm{HH}$ having any phone & $\begin{array}{c}8.1 \\
(338)\end{array}$ & $\begin{array}{c}32.1 \\
(1332)\end{array}$ & $\begin{array}{c}82.8 \\
(4524)\end{array}$ & $\begin{array}{c}89.3 \\
(2209)\end{array}$ & $\begin{array}{c}12.5 \\
(1872)\end{array}$ & $\begin{array}{c}39.2 \\
(5736)\end{array}$ & $\begin{array}{c}85.7 \\
(14759)\end{array}$ & $\begin{array}{c}91.0 \\
(8251)\end{array}$ & $\begin{array}{c}9.8 \\
(28359)\end{array}$ & $\begin{array}{c}36.0 \\
(114482)\end{array}$ & $\begin{array}{c}86.1 \\
(94697)\end{array}$ & $\begin{array}{c}90.5 \\
(211802)\end{array}$ \\
\hline Proportion of $\mathrm{HH}$ with any of 3 assets (TV, Fan, Refrigerator) & $\begin{array}{c}39.1 \\
(1593)\end{array}$ & $\begin{array}{c}48.7 \\
(1973)\end{array}$ & $\begin{array}{c}59.8 \\
(3281)\end{array}$ & $\begin{array}{c}84.5 \\
(2098)\end{array}$ & $\begin{array}{c}45.1 \\
(6125)\end{array}$ & $\begin{array}{c}53.7 \\
(7864)\end{array}$ & $\begin{array}{c}71.7 \\
(12346)\end{array}$ & $\begin{array}{c}86.6 \\
(7862)\end{array}$ & $\begin{array}{c}55.3 \\
(129424)\end{array}$ & $\begin{array}{c}50.7 \\
(160300)\end{array}$ & $\begin{array}{c}80.2 \\
(88773)\end{array}$ & $\begin{array}{c}77.1 \\
(177479)\end{array}$ \\
\hline Proportion of $\mathrm{HH}$ using clean fuel for cooking & $\begin{array}{l}11.1 \\
(459)\end{array}$ & $\begin{array}{c}4.9 \\
(184)\end{array}$ & $\begin{array}{l}10.1 \\
(557)\end{array}$ & $\begin{array}{l}22.4 \\
(523)\end{array}$ & $\begin{array}{c}15.0 \\
(2180)\end{array}$ & $\begin{array}{c}10.2 \\
(1483)\end{array}$ & $\begin{array}{c}23.9 \\
(4119)\end{array}$ & $\begin{array}{c}38.1 \\
(3290)\end{array}$ & $\begin{array}{c}17.3 \\
(39867)\end{array}$ & $\begin{array}{c}11.0 \\
(34399)\end{array}$ & $\begin{array}{c}36.4 \\
(40440)\end{array}$ & $\begin{array}{c}29.1 \\
(61097)\end{array}$ \\
\hline Proportion of $\mathrm{HH}$ uses a toilet facility & $\begin{array}{c}8.3 \\
(351)\end{array}$ & $\begin{array}{c}7.7 \\
(289)\end{array}$ & $\begin{array}{c}11.9 \\
(651)\end{array}$ & $\begin{array}{l}24.0 \\
(603)\end{array}$ & $\begin{array}{c}22.5 \\
(3435)\end{array}$ & $\begin{array}{c}28.3 \\
(4113)\end{array}$ & $\begin{array}{c}42.5 \\
(7349)\end{array}$ & $\begin{array}{c}53.5 \\
(4976)\end{array}$ & $\begin{array}{c}23.1 \\
(80258)\end{array}$ & $\begin{array}{c}33.9 \\
(108067)\end{array}$ & $\begin{array}{c}65.4 \\
(71926)\end{array}$ & $\begin{array}{c}51.0 \\
(121902)\end{array}$ \\
\hline Proportion of HH has Pakka/Semi Pakka house & $\begin{array}{c}79.4 \\
(3187)\end{array}$ & $\begin{array}{c}68.1 \\
(2835)\end{array}$ & $\begin{array}{c}84.7 \\
(4630)\end{array}$ & $\begin{array}{c}95.6 \\
(2370)\end{array}$ & $\begin{array}{c}87.0 \\
(11826)\end{array}$ & $\begin{array}{c}76.0 \\
(11148)\end{array}$ & $\begin{array}{c}87.1 \\
(15030)\end{array}$ & $\begin{array}{c}96.8 \\
(8744)\end{array}$ & $\begin{array}{c}66.1 \\
(161597)\end{array}$ & $\begin{array}{c}63.4 \\
(202848)\end{array}$ & $\begin{array}{c}84.2 \\
(92653)\end{array}$ & $\begin{array}{c}92.9 \\
(208486)\end{array}$ \\
\hline \multicolumn{13}{|l|}{ Marriage: } \\
\hline $\mathrm{N}$ & 1108 & 1191 & 1340 & 698 & 2958 & 2996 & 2906 & 2100 & 58537 & 67238 & 15145 & 69318 \\
\hline Proportion of women aged $18-24$ who were married below 18 years & $\begin{array}{l}74.4 \\
(819)\end{array}$ & $\begin{array}{l}70.7 \\
(846)\end{array}$ & $\begin{array}{l}49.5 \\
(667)\end{array}$ & $\begin{array}{l}25.3 \\
(171)\end{array}$ & $\begin{array}{c}59.8 \\
(1759)\end{array}$ & $\begin{array}{c}53.5 \\
(1607)\end{array}$ & $\begin{array}{c}39.5 \\
(1148)\end{array}$ & $\begin{array}{l}19.8 \\
(394)\end{array}$ & $\begin{array}{c}59.6 \\
(32801)\end{array}$ & $\begin{array}{c}50.7 \\
(34416)\end{array}$ & $\begin{array}{l}32.9 \\
(4924)\end{array}$ & $\begin{array}{c}22.7 \\
(14474)\end{array}$ \\
\hline \multicolumn{13}{|l|}{ SRH: } \\
\hline $\mathbf{N}$ & 1108 & 1191 & 1340 & 698 & 2958 & 2996 & 2906 & 2100 & 58537 & 67238 & 15145 & 69318 \\
\hline $\begin{array}{l}\text { Proportion of women aged } 18-24 \text { who were married }<18 \text { years and } \\
\text { co-habiting }<18 \text { years }\end{array}$ & $\begin{array}{l}74.4 \\
(819)\end{array}$ & $\begin{array}{l}68.0 \\
(817)\end{array}$ & $\begin{array}{l}45.4 \\
(613)\end{array}$ & $\begin{array}{l}23.9 \\
(161)\end{array}$ & $\begin{array}{c}59.8 \\
(1759)\end{array}$ & $\begin{array}{c}52.2 \\
(1569)\end{array}$ & $\begin{array}{c}36.7 \\
(1067)\end{array}$ & $\begin{array}{l}18.6 \\
(371)\end{array}$ & $\begin{array}{c}59.6 \\
(32801)\end{array}$ & $\begin{array}{c}44.8 \\
(30345)\end{array}$ & $\begin{array}{c}31.2 \\
(4670)\end{array}$ & $\begin{array}{c}20.6 \\
(13000)\end{array}$ \\
\hline $\mathrm{N}$ & 430 & 361 & 280 & 396 & 783 & 609 & 445 & 1160 & 13533 & 14023 & 1698 & 42565 \\
\hline $\begin{array}{l}\text { Proportion of married girls aged < } 19 \text { years pregnant or have } \\
\text { children }\end{array}$ & $\begin{array}{l}59.4 \\
(252)\end{array}$ & $\begin{array}{l}52.9 \\
(197)\end{array}$ & $\begin{array}{l}50.4 \\
(143)\end{array}$ & $\begin{array}{l}4.1 \\
(16)\end{array}$ & $\begin{array}{l}53.5 \\
(443)\end{array}$ & $\begin{array}{l}51.4 \\
(313)\end{array}$ & $\begin{array}{l}51.1 \\
(230)\end{array}$ & $\begin{array}{l}5.0 \\
(49)\end{array}$ & $\begin{array}{l}50.8 \\
(6760)\end{array}$ & $\begin{array}{l}48.0 \\
(6761)\end{array}$ & $\begin{array}{l}48.5 \\
(826)\end{array}$ & $\begin{array}{c}4.9 \\
(1823)\end{array}$ \\
\hline $\mathrm{N}$ & 2962 & 3830 & 4948 & 2032 & 9601 & 12748 & 14482 & 6938 & 192477 & 271004 & 83787 & 193625 \\
\hline Proportion of Mothers who are literate & $\begin{array}{l}30.4 \\
(946)\end{array}$ & $\begin{array}{c}40.1 \\
(1503)\end{array}$ & $\begin{array}{c}45.9 \\
(2273)\end{array}$ & $\begin{array}{c}49.3 \\
(1001)\end{array}$ & $\begin{array}{c}46.7 \\
(4706)\end{array}$ & $\begin{array}{c}56.8 \\
(7228)\end{array}$ & $\begin{array}{c}62.5 \\
(9055)\end{array}$ & $\begin{array}{c}66.2 \\
(4574)\end{array}$ & $\begin{array}{c}45.4 \\
(88892)\end{array}$ & $\begin{array}{c}50.7 \\
(136045)\end{array}$ & $\begin{array}{c}69.5 \\
(58359)\end{array}$ & $\begin{array}{c}60.9 \\
(118019)\end{array}$ \\
\hline \multicolumn{13}{|l|}{ Education: } \\
\hline $\mathbf{N}$ & 849 & 809 & 908 & 372 & 2322 & 2328 & 2254 & 1079 & 49854 & 62188 & 12381 & 35341 \\
\hline Proportion of girls aged 12-14 entered into secondary school & $\begin{array}{l}16.6 \\
(135)\end{array}$ & $\begin{array}{l}21.6 \\
(172)\end{array}$ & $\begin{array}{l}26.1 \\
(238)\end{array}$ & $\begin{array}{l}31.2 \\
(113)\end{array}$ & $\begin{array}{l}26.3 \\
(596)\end{array}$ & $\begin{array}{l}28.1 \\
(654)\end{array}$ & $\begin{array}{l}39.1 \\
(886)\end{array}$ & $\begin{array}{l}36.1 \\
(397)\end{array}$ & $\begin{array}{c}26.7 \\
(9641)\end{array}$ & $\begin{array}{c}22.1 \\
(13509)\end{array}$ & $\begin{array}{c}45.6 \\
(5653)\end{array}$ & $\begin{array}{c}29.5 \\
(10386)\end{array}$ \\
\hline
\end{tabular}




\begin{tabular}{|c|c|c|c|c|c|c|c|c|c|c|c|c|}
\hline $\mathrm{N}$ & 861 & 828 & 860 & 272 & 2410 & 2302 & 2193 & 911 & 45469 & 51413 & 12193 & 33076 \\
\hline Proportion of girls aged 15-17 completed secondary education & $\begin{array}{l}18.8 \\
(175)\end{array}$ & $\begin{array}{l}27.0 \\
(213)\end{array}$ & $\begin{array}{l}38.4 \\
(331)\end{array}$ & $\begin{array}{l}45.1 \\
(120)\end{array}$ & $\begin{array}{l}27.7 \\
(693)\end{array}$ & $\begin{array}{l}38.7 \\
(889)\end{array}$ & $\begin{array}{c}54.6 \\
(1196)\end{array}$ & $\begin{array}{l}60.8 \\
(552)\end{array}$ & $\begin{array}{c}25.2 \\
(8816)\end{array}$ & $\begin{array}{c}25.5 \\
(12935)\end{array}$ & $\begin{array}{c}59.5 \\
(7304)\end{array}$ & $\begin{array}{c}40.0 \\
(12812)\end{array}$ \\
\hline $\mathrm{N}$ & 1030 & 1030 & 1188 & 346 & 3196 & 2928 & 2733 & 1043 & 54444 & 59795 & 14405 & 34811 \\
\hline $\begin{array}{l}\text { Proportion of girls aged } 18-20 \text { completed higher-secondary } \\
\text { education }\end{array}$ & $\begin{array}{c}9.3 \\
(100)\end{array}$ & $\begin{array}{l}12.0 \\
(117)\end{array}$ & $\begin{array}{l}20.9 \\
(252)\end{array}$ & $\begin{array}{l}33.8 \\
(119)\end{array}$ & $\begin{array}{l}12.7 \\
(457)\end{array}$ & $\begin{array}{l}20.8 \\
(606)\end{array}$ & $\begin{array}{l}34.9 \\
(964)\end{array}$ & $\begin{array}{c}44.5 \\
(469)\end{array}$ & $\begin{array}{c}14.7 \\
(6671)\end{array}$ & $\begin{array}{c}17.4 \\
(10288)\end{array}$ & $\begin{array}{c}46.7 \\
(6804)\end{array}$ & $\begin{array}{c}36.0 \\
(12378)\end{array}$ \\
\hline
\end{tabular}

DLHS - District Level Household and Facility Surveys, NFHS - National Family Health Survey, HH - Household, SRH - Sexual and Reproductive Health "9 districts include - Bidar, Gulbarga, Bijapur, Yadgir, Bagalkot, Raichur, Gadag, Koppal and Bellary.

$\uparrow$ Data not collected from 8 poorly performing States in India (as data from these States were collected in a different survey). 


\section{Table 3. Profile of rural Scheduled Caste/Scheduled Tribe girls}

\begin{tabular}{|c|c|c|c|c|c|c|c|c|c|c|c|c|}
\hline \multirow{4}{*}{ Indicators } & \multicolumn{4}{|c|}{$\begin{array}{l}\text { Northern Karnataka Districts } \\
\text { (9 districts) }{ }^{*}\end{array}$} & \multicolumn{4}{|c|}{ Karnataka } & \multicolumn{4}{|c|}{ India } \\
\hline & DLHSII & $\begin{array}{c}\text { DLHS } \\
\text { III }\end{array}$ & $\begin{array}{l}\text { DLHS } \\
\text { IV }\end{array}$ & $\begin{array}{l}\text { NFHS } \\
\text { IV }\end{array}$ & DLHS II & $\begin{array}{l}\text { DLHS } \\
\text { III }\end{array}$ & $\begin{array}{l}\text { DLHS } \\
\text { IV }+\end{array}$ & $\begin{array}{l}\text { NFHS } \\
\text { IV }\end{array}$ & DLHS II & DLHS III & $\begin{array}{l}\text { DLHS } \\
\text { IV } \dagger\end{array}$ & NFHS IV \\
\hline & $2002 / 3$ & $2007 / 8$ & $2012 / 3$ & $2015 / 6$ & $2002 / 3$ & $2007 / 8$ & $2012 / 3$ & $2015 / 6$ & $2002 / 3$ & $2007 / 8$ & $2012 / 3$ & $2015 / 6$ \\
\hline & $\%(N)$ & $\%(N)$ & $\%(N)$ & $\%(N)$ & $\%(N)$ & $\%(N)$ & $\%(N)$ & $\%(N)$ & $\%(N)$ & $\%(N)$ & $\%(N)$ & $\%(N)$ \\
\hline \multicolumn{13}{|l|}{ Economic: } \\
\hline $\mathrm{N}$ & 1791 & 2271 & 3475 & 1922 & 5369 & 6226 & 9328 & 5382 & 159480 & 227207 & 107869 & 174913 \\
\hline Proportion of $\mathrm{HH}$ with electricity & $\begin{array}{c}74.9 \\
(1342)\end{array}$ & $\begin{array}{c}85.8 \\
(1940)\end{array}$ & $\begin{array}{c}92.9 \\
(3222)\end{array}$ & $\begin{array}{c}96.7 \\
(1863)\end{array}$ & $\begin{array}{c}76.0 \\
(4035)\end{array}$ & $\begin{array}{l}81.7 \\
(5086)\end{array}$ & $\begin{array}{c}93.3 \\
(8699)\end{array}$ & $\begin{array}{c}95.7 \\
(5132)\end{array}$ & $\begin{array}{c}56.5 \\
(81352)\end{array}$ & $\begin{array}{c}56.4 \\
(125151)\end{array}$ & $\begin{array}{c}90.8 \\
(97692)\end{array}$ & $\begin{array}{c}80.4 \\
(144231)\end{array}$ \\
\hline Proportion of $\mathrm{HH}$ having any phone & $2.8(54)$ & $\begin{array}{l}20.0 \\
(463)\end{array}$ & $\begin{array}{c}76.9 \\
(2673)\end{array}$ & $\begin{array}{c}83.7 \\
(1592)\end{array}$ & $\begin{array}{c}3.8 \\
(218)\end{array}$ & $\begin{array}{c}22.4 \\
(1392)\end{array}$ & $\begin{array}{c}78.9 \\
(7366)\end{array}$ & $\begin{array}{c}84.1 \\
(4511)\end{array}$ & $\begin{array}{c}3.2 \\
(8462)\end{array}$ & $\begin{array}{c}19.3 \\
(43356)\end{array}$ & $\begin{array}{c}78.0 \\
(84346)\end{array}$ & $\begin{array}{c}81.8 \\
(143176)\end{array}$ \\
\hline Proportion of $\mathrm{HH}$ with any of 3 assets (TV, Fan, Refrigerator) & $\begin{array}{l}20.6 \\
(385)\end{array}$ & $\begin{array}{l}33.6 \\
(755)\end{array}$ & $\begin{array}{c}51.2 \\
(1780)\end{array}$ & $\begin{array}{c}77.6 \\
(1484)\end{array}$ & $\begin{array}{c}24.4 \\
(1296)\end{array}$ & $\begin{array}{c}35.7 \\
(2221)\end{array}$ & $\begin{array}{c}62.2 \\
(5789)\end{array}$ & $\begin{array}{c}77.7 \\
(4150)\end{array}$ & $\begin{array}{c}34.2 \\
(48337)\end{array}$ & $\begin{array}{c}34.4 \\
(77350)\end{array}$ & $\begin{array}{c}65.5 \\
(70663)\end{array}$ & $\begin{array}{c}65.4 \\
(108548)\end{array}$ \\
\hline Proportion of $\mathrm{HH}$ using clean fuel for cooking & $4.3(80)$ & $0.8(20)$ & $\begin{array}{c}3.7 \\
(126)\end{array}$ & $\begin{array}{c}11.1 \\
(188)\end{array}$ & $\begin{array}{c}5.8 \\
(330)\end{array}$ & $\begin{array}{c}2.5 \\
(151)\end{array}$ & $\begin{array}{c}11.8 \\
(1091)\end{array}$ & $\begin{array}{c}21.7 \\
(1104)\end{array}$ & $\begin{array}{c}6.7 \\
(16616)\end{array}$ & $\begin{array}{c}5.9 \\
(12859)\end{array}$ & $\begin{array}{c}19.8 \\
(21446)\end{array}$ & $\begin{array}{c}15.0 \\
(25776)\end{array}$ \\
\hline Proportion of $\mathrm{HH}$ uses a toilet facility & $\begin{array}{c}9.2 \\
(173)\end{array}$ & $2.6(53)$ & $\begin{array}{c}4.6 \\
(159)\end{array}$ & $\begin{array}{l}18.3 \\
(359)\end{array}$ & $\begin{array}{l}11.3 \\
(652)\end{array}$ & $\begin{array}{l}10.7 \\
(658)\end{array}$ & $\begin{array}{c}21.9 \\
(2042)\end{array}$ & $\begin{array}{c}35.9 \\
(1997)\end{array}$ & $\begin{array}{c}11.3 \\
(45986)\end{array}$ & $\begin{array}{c}32.4 \\
(70207)\end{array}$ & $\begin{array}{c}62.6 \\
(69047)\end{array}$ & $\begin{array}{c}33.7 \\
(79128)\end{array}$ \\
\hline Proportion of HH has Pakka/Semi Pakka house & $\begin{array}{c}70.6 \\
(1267)\end{array}$ & $\begin{array}{c}55.2 \\
(1231)\end{array}$ & $\begin{array}{c}76.7 \\
(2670)\end{array}$ & $\begin{array}{c}91.8 \\
(1747)\end{array}$ & $\begin{array}{c}77.4 \\
(4210)\end{array}$ & $\begin{array}{c}63.2 \\
(3932)\end{array}$ & $\begin{array}{c}78.6 \\
(7341)\end{array}$ & $\begin{array}{c}93.4 \\
(4981)\end{array}$ & $\begin{array}{c}47.9 \\
(66009)\end{array}$ & $\begin{array}{c}41.0 \\
(91623)\end{array}$ & $\begin{array}{c}64.1 \\
(68557)\end{array}$ & $\begin{array}{c}88.9 \\
(146233)\end{array}$ \\
\hline \multicolumn{13}{|l|}{ Marriage: } \\
\hline $\mathrm{N}$ & 533 & 674 & 943 & 600 & 1430 & 1525 & 1933 & 1496 & 33980 & 44001 & 14786 & 49370 \\
\hline Proportion of women aged $18-24$ who were married below 18 years & $\begin{array}{l}83.6 \\
(445)\end{array}$ & $\begin{array}{l}82.4 \\
(555)\end{array}$ & $\begin{array}{l}55.6 \\
(525)\end{array}$ & $\begin{array}{l}28.3 \\
(174)\end{array}$ & $\begin{array}{c}72.8 \\
(1051)\end{array}$ & $\begin{array}{c}68.4 \\
(1043)\end{array}$ & $\begin{array}{l}46.1 \\
(895)\end{array}$ & $\begin{array}{l}24.7 \\
(364)\end{array}$ & $\begin{array}{c}67.9 \\
(20928)\end{array}$ & $\begin{array}{c}53.3 \\
(23698)\end{array}$ & $\begin{array}{c}35.3 \\
(5176)\end{array}$ & $\begin{array}{c}25.7 \\
(10941)\end{array}$ \\
\hline \multicolumn{13}{|l|}{ SRH: } \\
\hline $\mathbf{N}$ & 533 & 674 & 943 & 600 & 1430 & 1525 & 1933 & 1496 & 33980 & 44001 & 14786 & 49370 \\
\hline $\begin{array}{l}\text { Proportion of women aged } 18-24 \text { who were married }<18 \text { years and } \\
\text { co-habiting }<18 \text { years }\end{array}$ & $\begin{array}{l}83.6 \\
(445)\end{array}$ & $\begin{array}{l}81.5 \\
(549)\end{array}$ & $\begin{array}{l}52.4 \\
(496)\end{array}$ & $\begin{array}{l}27.6 \\
(168)\end{array}$ & $\begin{array}{c}72.8 \\
(1051)\end{array}$ & $\begin{array}{c}67.9 \\
(1036)\end{array}$ & $\begin{array}{l}43.0 \\
(836)\end{array}$ & $\begin{array}{l}24.1 \\
(353)\end{array}$ & $\begin{array}{c}67.9 \\
(20928)\end{array}$ & $\begin{array}{c}49.2 \\
(21824)\end{array}$ & $\begin{array}{c}33.3 \\
(4892)\end{array}$ & $\begin{array}{c}24.1 \\
(10256)\end{array}$ \\
\hline $\mathrm{N}$ & 275 & 271 & 209 & 263 & 512 & 441 & 317 & 734 & 8493 & 8795 & 1648 & 29969 \\
\hline Proportion of married girls aged < 19 years pregnant or have children & $\begin{array}{l}60.8 \\
(167)\end{array}$ & $\begin{array}{c}60.2 \\
(163)\end{array}$ & $\begin{array}{l}58.5 \\
(122)\end{array}$ & $6.4(18)$ & $\begin{array}{l}61.3 \\
(320)\end{array}$ & $\begin{array}{l}57.3 \\
(253)\end{array}$ & $\begin{array}{l}54.8 \\
(174)\end{array}$ & $7.0(47)$ & $\begin{array}{c}56.3 \\
(4711)\end{array}$ & $\begin{array}{c}46.9 \\
(4144)\end{array}$ & $\begin{array}{l}50.8 \\
(854)\end{array}$ & $\begin{array}{c}6.1 \\
(1581)\end{array}$ \\
\hline $\mathbf{N}$ & 1359 & 2113 & 3217 & 1574 & 3909 & 5516 & 8168 & 4347 & 113315 & 172660 & 79006 & 134719 \\
\hline Proportion of Mothers who are literate & $\begin{array}{l}13.5 \\
(179)\end{array}$ & $\begin{array}{l}20.9 \\
(435)\end{array}$ & $\begin{array}{l}26.5 \\
(864)\end{array}$ & $\begin{array}{l}37.1 \\
(575)\end{array}$ & $\begin{array}{l}25.4 \\
(977)\end{array}$ & $\begin{array}{c}32.2 \\
(1775)\end{array}$ & $\begin{array}{c}41.7 \\
(3413)\end{array}$ & $\begin{array}{c}50.8 \\
(2189)\end{array}$ & $\begin{array}{c}26.1 \\
(38180)\end{array}$ & $\begin{array}{c}39.4 \\
(66824)\end{array}$ & $\begin{array}{c}56.3 \\
(45169)\end{array}$ & $\begin{array}{c}48.6 \\
(69669)\end{array}$ \\
\hline \multicolumn{13}{|l|}{ Education: } \\
\hline $\mathbf{N}$ & 430 & 568 & 665 & 304 & 1086 & 1224 & 1501 & 720 & 30741 & 43909 & 14477 & 27587 \\
\hline Proportion of girls aged 12-14 entered into secondary school & $5.6(27)$ & $\begin{array}{l}14.9 \\
(84)\end{array}$ & $\begin{array}{l}17.9 \\
(122)\end{array}$ & $\begin{array}{l}21.4 \\
(62)\end{array}$ & $\begin{array}{l}19.1 \\
(202)\end{array}$ & $\begin{array}{l}21.9 \\
(268)\end{array}$ & $\begin{array}{l}31.4 \\
(475)\end{array}$ & $\begin{array}{l}29.7 \\
(209)\end{array}$ & $\begin{array}{c}21.5 \\
(3928)\end{array}$ & $\begin{array}{c}15.5 \\
(6741)\end{array}$ & $\begin{array}{c}33.6 \\
(4823)\end{array}$ & $\begin{array}{c}25.6 \\
(6517)\end{array}$ \\
\hline
\end{tabular}




\begin{tabular}{|c|c|c|c|c|c|c|c|c|c|c|c|c|}
\hline $\mathbf{N}$ & 408 & 483 & 589 & 205 & 1031 & 1069 & 1319 & 551 & 27736 & 33906 & 13641 & 23999 \\
\hline Proportion of girls aged 15-17 completed secondary education & $7.9(34)$ & $\begin{array}{l}18.2 \\
(87)\end{array}$ & $\begin{array}{l}25.1 \\
(152)\end{array}$ & $\begin{array}{l}36.7 \\
(77)\end{array}$ & $\begin{array}{c}13.9 \\
(155)\end{array}$ & $\begin{array}{l}25.2 \\
(270)\end{array}$ & $\begin{array}{l}40.2 \\
(533)\end{array}$ & $\begin{array}{l}47.9 \\
(263)\end{array}$ & $\begin{array}{c}15.4 \\
(3158)\end{array}$ & $\begin{array}{l}15.8 \\
(5314)\end{array}$ & $\begin{array}{c}40.4 \\
(5477)\end{array}$ & $\begin{array}{c}30.5 \\
(6519)\end{array}$ \\
\hline$N$ & 431 & 574 & 852 & 281 & 1328 & 1426 & 1763 & 697 & 33123 & 41452 & 15381 & 25120 \\
\hline $\begin{array}{l}\text { Proportion of girls aged } 18-20 \text { completed higher-secondary } \\
\text { education }\end{array}$ & $3.6(16)$ & $6.0(33)$ & $\begin{array}{r}12.9 \\
(113)\end{array}$ & $\begin{array}{l}17.9 \\
(49)\end{array}$ & $6.2(81)$ & $\begin{array}{l}10.0 \\
(143)\end{array}$ & $\begin{array}{l}21.6 \\
(389)\end{array}$ & $\begin{array}{l}29.1 \\
(204)\end{array}$ & $\begin{array}{c}7.8 \\
(2322)\end{array}$ & $\begin{array}{c}9.3 \\
(3861)\end{array}$ & $\begin{array}{c}28.6 \\
(4409)\end{array}$ & $\begin{array}{c}23.7 \\
(5444)\end{array}$ \\
\hline
\end{tabular}

DLHS - District Level Household and Facility Surveys, NFHS - National Family Health Survey, HH - Household, SRH - Sexual and Reproductive Health

*9 districts include - Bidar, Gulbarga, Bijapur, Yadgir, Bagalkot, Raichur, Gadag, Koppal and Bellary.

$\dagger$ Data not collected from 8 poorly performing States in India (as data from these States were collected in a different survey). 
eas in northern Karnataka, Karnataka state and India. Educational outcomes have improved substantially among girls, with more than double the proportion of girls living in rural northern Karnataka starting secondary school (12.8\% vs. 26.9\%), completing secondary school (15.3\% vs. $41.5 \%)$, and completing higher secondary education (7.6\% vs. $26.6 \%)$ in 2015/6 compared with 2002/3 (Table 1). However, in 2015/ 6, rural girls from SC/ST households were still less likely to start secondary school (21.4\% vs. $31.2 \%)$, or complete secondary (36.7\% vs. $45.1 \%$ ) or higher-secondary education (17.9\% vs. 33.8\%), compared with rural girls from non-SC/ ST households (Tables $\underline{2}$ and $\underline{3}$ ). These overall improvements in educational attainment, and disparities based on caste, were also seen across Karnataka state and India.

Similarly, between 2002/3 and 2015/6, there was a dramatic increase in the proportion of boys living in rural areas in northern Karnataka who (i) entered into secondary school (16.7\% vs. $25.5 \%$ ), (ii) completed secondary school (23.0\% vs. 41.0\%), and (iii) completed higher-secondary education (15.0\% vs. 38.4\%) (Table 4). Boys from non-SC/ST families had better secondary and higher-secondary completion rates than boys from SC/ST backgrounds (Table 5 and Table 6). These improvements and patterns were also seen among boys living in rural areas across Karnataka state and all India.

Of note, by $2015 / 6$ there was parity in secondary school entry (24.5\% vs. $26.9 \%)$ and completion (41.0\% vs. $41.5 \%)$ between rural boys and girls in northern Karnataka, but boys were still more likely to complete higher secondary school education compared with girls (38.4\% vs. 26.6\%). Similar gender parity in secondary school educational attainment was also seen in the 2015/16 data from all of Karnataka and across India. However, for both girls and boys, rates of secondary school entry and completion remained far lower in northern Karnataka compared with Karnataka state, but rates in northern Karnataka are comparable to allIndia estimates (Table 1 and Table 4).

\section{ASSOCIATIONS BETWEEN SOCIAL FACTORS, MARRIAGE AND EDUCATION AMONG RURAL ADOLESCENT GIRLS}

To identify which girls remain most at risk of child marriage, we conducted univariate regression analyses using NFHS-4 data (2015/6) from rural Karnataka state (Table 7). Compared with girls who were married aged 20-24 years, girls who were married $<18$ years were significantly more likely to be from the poorest wealth quintile and to be illiterate, with the odds increasing with decreasing age at marriage (Table 7). In addition, there was evidence that marriage aged 16-17 years was more likely among girls from SC/ ST castes, but there was no evidence of caste differences in marriage rates for girls married $<16$ years old. When we repeated these analyses using data from all rural India, we found significant associations between child marriage and SC/ST caste, poverty and illiteracy (Table 7).

To identify which girls remain most at risk of not completing secondary school education, we again conducted regression analyses using NFHS-4 data. Across rural Karnataka state, among girls aged $12-14$ years, SC/ST caste and being from the poorest quintile were significantly associated with not starting secondary school. Among girls aged 15-17 years and 18-20 years, SC/ST caste, poverty and marriage were significantly associated with not completing secondary school or higher-secondary school, respectively (Table 8). These patterns were also found when we repeated these analyses using data from all rural India (Table 8).

\section{DISCUSSION}

In this study, we conducted an ecological analysis of preexisting, nationally representative datasets and found large improvements in household economic indicators and secondary educational attainment, and large reductions in child marriage and early child-bearing rates over the past 15 years, at the district, state and national levels. Not only that, we also found evidence of clustered deprivations, whereby adolescent girls living in rural areas from the lowest castes (SC/ST) and the poorest families, continue to be the most at risk of secondary school non-completion and early marriage. This study adds to the evidence base by using nationally representative data, including those from the most recent survey (2015/6), to document these trends, and to examine intersecting vulnerabilities of the most marginalised girls. These trends in child marriage and secondary school retention have also been noted in other LMICs globally. ${ }^{7,16,29}$ The findings will be useful for education and health policy makers and implementers seeking to identify those individuals and families who continue to be most at risk, so as to inform new policies and programming to achieve the SDG targets of leaving no one behind.

The improvements in educational attainment over the past 14 years, and the reductions in child marriage and early child-bearing rates, were seen among rural households in northern Karnataka as well as across Karnataka state and India and help explain the low rates of child marriage and secondary school drop-out seen at trial end in the Samata trial (26). Nonetheless, secondary school nonentry and child marriage rates remain highest among SC/ ST girls living in rural northern Karnataka, compared with all of Karnataka and India, suggesting northern Karnataka remains disadvantaged. There have also been substantial economic improvements for rural households across India, with more households living in semi-permanent or permanent dwellings, and increased access to electricity, clean fuel, toilet facilities, and phones. Caste disparities persist with regard to economic indicators, education and marriage rates, and higher secondary school (16-18 years) completion, but gender parity in lower secondary school enrolment and completion has been achieved. This evidence suggests that sustained political will, coupled with legislative and policy changes, appears to have benefited rural young people, within relatively short time-frames.

Despite these successes, there remains a sizeable population still at risk of poor educational outcomes, child marriage and early child-bearing. The current analysis suggests that it is young people (aged 12-20 years) from the poorest households and the lowest castes (SC/ST) that are most at risk of not starting or completing secondary school, marrying early and bearing children during adolescence. Identifying and supporting these girls-those who experience multiple forms of clustered disadvantage-will be key to India achieving its Sustainable Development Goal (SDG) aspira- 


\section{Table 4. Profile of all rural boys}

\begin{tabular}{|c|c|c|c|c|c|c|c|c|c|c|c|c|}
\hline \multirow{4}{*}{ Indicators } & \multicolumn{4}{|c|}{$\begin{array}{l}\text { Northern Karnataka Districts } \\
\text { (9 districts)* }\end{array}$} & \multicolumn{4}{|c|}{ Karnataka } & \multicolumn{4}{|c|}{ India } \\
\hline & $\begin{array}{l}\text { DLHS } \\
\text { II }\end{array}$ & $\begin{array}{l}\text { DLHS } \\
\text { III }\end{array}$ & $\begin{array}{l}\text { DLHS } \\
\text { IV }\end{array}$ & $\begin{array}{l}\text { NFHS } \\
\text { IV }\end{array}$ & $\begin{array}{l}\text { DLHS } \\
\text { II }\end{array}$ & $\begin{array}{c}\text { DLHS } \\
\text { III }\end{array}$ & $\begin{array}{l}\text { DLHS } \\
\text { IV }\end{array}$ & $\begin{array}{l}\text { NFHS } \\
\text { IV }\end{array}$ & DLHSII & $\begin{array}{c}\text { DLHS } \\
\text { III }\end{array}$ & $\begin{array}{l}\text { DLHS } \\
\text { IV } †\end{array}$ & $\begin{array}{l}\text { NFHS } \\
\text { IV }\end{array}$ \\
\hline & $2002 / 3$ & $2007 / 8$ & $2012 / 3$ & $2015 / 6$ & $2002 / 3$ & $2007 / 8$ & $2012 / 3$ & $2015 / 6$ & $2002 / 3$ & $2007 / 8$ & $2012 / 3$ & $2015 / 6$ \\
\hline & $\%(N)$ & $\%(N)$ & $\%(N)$ & $\%(N)$ & $\%(N)$ & $\%(N)$ & $\%(N)$ & $\%(N)$ & $\%(N)$ & $\%(N)$ & $\%(N)$ & $\%(N)$ \\
\hline \multicolumn{13}{|l|}{ Education: } \\
\hline$N$ & 1320 & 1405 & 1591 & 653 & 3607 & 3615 & 3696 & 1751 & 86247 & 107262 & 28750 & 65380 \\
\hline $\begin{array}{l}\text { Proportion of boys aged } 12-14 \\
\text { entered into secondary school }\end{array}$ & $\begin{array}{l}16.7 \\
(225)\end{array}$ & $\begin{array}{l}21.8 \\
(299)\end{array}$ & $\begin{array}{l}23.9 \\
(379)\end{array}$ & $\begin{array}{l}24.5 \\
(155)\end{array}$ & $\begin{array}{l}23.2 \\
(841)\end{array}$ & $\begin{array}{l}25.5 \\
(922)\end{array}$ & $\begin{array}{c}32.5 \\
(1202)\end{array}$ & $\begin{array}{c}33.2 \\
(581)\end{array}$ & $\begin{array}{c}24.8 \\
(16111)\end{array}$ & $\begin{array}{c}19.8 \\
(21092)\end{array}$ & $\begin{array}{c}36.2 \\
(10327)\end{array}$ & $\begin{array}{c}26.6 \\
(16728)\end{array}$ \\
\hline$N$ & 1088 & 1267 & 1511 & 639 & 3166 & 3358 & 3625 & 1710 & 74257 & 95705 & 28116 & 60272 \\
\hline $\begin{array}{l}\text { Proportion of boys aged } 15-17 \\
\text { completed secondary education }\end{array}$ & $\begin{array}{l}23.3 \\
(256)\end{array}$ & $\begin{array}{l}30.1 \\
(395)\end{array}$ & $\begin{array}{l}34.0 \\
(513)\end{array}$ & $\begin{array}{l}41.0 \\
(247)\end{array}$ & $\begin{array}{l}27.3 \\
(861)\end{array}$ & $\begin{array}{c}37.2 \\
(1250)\end{array}$ & $\begin{array}{c}45.0 \\
(1639)\end{array}$ & $\begin{array}{l}48.4 \\
(813)\end{array}$ & $\begin{array}{c}25.3 \\
(14589)\end{array}$ & $\begin{array}{c}23.6 \\
(22520)\end{array}$ & $\begin{array}{c}48.0 \\
(13440)\end{array}$ & $\begin{array}{c}35.6 \\
(20089)\end{array}$ \\
\hline$N$ & 1324 & 1405 & 1764 & 710 & 3867 & 3804 & 4163 & 1858 & 81261 & 103323 & 29753 & 57503 \\
\hline $\begin{array}{l}\text { Proportion of boys aged 18-20 } \\
\text { completed higher-secondary } \\
\text { education }\end{array}$ & $\begin{array}{l}15.0 \\
(196)\end{array}$ & $\begin{array}{l}22.8 \\
(309)\end{array}$ & $\begin{array}{l}31.3 \\
(551)\end{array}$ & $\begin{array}{l}38.4 \\
(259)\end{array}$ & $\begin{array}{l}17.4 \\
(670)\end{array}$ & $\begin{array}{l}25.7 \\
(976)\end{array}$ & $\begin{array}{c}36.9 \\
(1542)\end{array}$ & $\begin{array}{l}39.8 \\
(737)\end{array}$ & $\begin{array}{c}18.9 \\
(12915)\end{array}$ & $\begin{array}{c}19.9 \\
(20568)\end{array}$ & $\begin{array}{c}41.5 \\
(12298)\end{array}$ & $\begin{array}{c}36.8 \\
(19457)\end{array}$ \\
\hline
\end{tabular}

DLHS - D strict Level Household and Facility Surveys, NFHS - National Family Health Survey

"9 districts include - Bidar, Gulbarga, Bijapur, Yadgir, Bagalkot, Raichur, Gadag, Koppal and Bellary.

†Data not collected from 8 poorly performing States in India (as data from these States were collected in a different survey). 
Table 5. Profile of rural non- Scheduled Caste /Scheduled Tribe boys

\begin{tabular}{|c|c|c|c|c|c|c|c|c|c|c|c|c|}
\hline \multirow{4}{*}{ Indicators } & \multicolumn{4}{|c|}{ Northern Karnataka Districts (9 districts)* } & \multicolumn{4}{|c|}{ Karnataka } & \multicolumn{4}{|c|}{ India } \\
\hline & $\begin{array}{l}\text { DLHS } \\
\text { II }\end{array}$ & $\begin{array}{l}\text { DLHS } \\
\text { III }\end{array}$ & $\begin{array}{l}\text { DLHS } \\
\text { IV }\end{array}$ & $\begin{array}{l}\text { NFHS } \\
\text { IV }\end{array}$ & $\begin{array}{l}\text { DLHS } \\
\text { II }\end{array}$ & $\begin{array}{l}\text { DLHS } \\
\text { III }\end{array}$ & $\begin{array}{l}\text { DLHS } \\
\text { IV } †\end{array}$ & $\begin{array}{l}\text { NFHS } \\
\text { IV }\end{array}$ & DLHS II & $\begin{array}{l}\text { DLHS } \\
\text { III }\end{array}$ & $\begin{array}{l}\text { DLHS } \\
\text { IV } †\end{array}$ & $\begin{array}{l}\text { NFHS } \\
\text { IV }\end{array}$ \\
\hline & $2002 / 3$ & $2007 / 8$ & $2012 / 3$ & $2015 / 6$ & $2002 / 3$ & $2007 / 8$ & $2012 / 3$ & $2015 / 6$ & $2002 / 3$ & $2007 / 8$ & $2012 / 3$ & $2015 / 6$ \\
\hline & $\%(N)$ & $\%(N)$ & $\%(N)$ & $\%(N)$ & $\%(N)$ & $\%(N)$ & $\%(N)$ & $\%(N)$ & $\%(N)$ & $\%(N)$ & $\%(N)$ & $\%(N)$ \\
\hline \multicolumn{13}{|l|}{ Education: } \\
\hline $\mathrm{N}$ & 881 & 851 & 895 & 324 & 2507 & 2441 & 2235 & 1003 & 53546 & 63613 & 13621 & 37517 \\
\hline $\begin{array}{l}\text { Proportion of boys aged } 12-14 \\
\text { entered into secondary school }\end{array}$ & $\begin{array}{l}18.0 \\
(165)\end{array}$ & $\begin{array}{r}21.6 \\
(180)\end{array}$ & $\begin{array}{l}26.9 \\
(238)\end{array}$ & $\begin{array}{l}23.8 \\
(76)\end{array}$ & $\begin{array}{l}24.3 \\
(619)\end{array}$ & $\begin{array}{l}26.7 \\
(651)\end{array}$ & $\begin{array}{l}35.4 \\
(790)\end{array}$ & $\begin{array}{l}33.9 \\
(344)\end{array}$ & $\begin{array}{c}26.5 \\
(11287)\end{array}$ & $\begin{array}{c}22.1 \\
(13874)\end{array}$ & $\begin{array}{c}42.2 \\
(5768)\end{array}$ & $\begin{array}{c}27.7 \\
(10410)\end{array}$ \\
\hline $\mathbf{N}$ & 729 & 791 & 888 & 364 & 2248 & 2278 & 2243 & 1044 & 46086 & 56663 & 13423 & 34778 \\
\hline $\begin{array}{l}\text { Proportion of boys aged } 15-17 \\
\text { completed secondary education }\end{array}$ & $\begin{array}{r}26.6 \\
(198)\end{array}$ & $\begin{array}{l}33.1 \\
(273)\end{array}$ & $\begin{array}{l}37.1 \\
(331)\end{array}$ & $\begin{array}{l}43.7 \\
(153)\end{array}$ & $\begin{array}{l}30.7 \\
(686)\end{array}$ & $\begin{array}{l}39.7 \\
(905)\end{array}$ & $\begin{array}{c}49.4 \\
(1113)\end{array}$ & $\begin{array}{l}50.9 \\
(522)\end{array}$ & $\begin{array}{c}29.0 \\
(10557)\end{array}$ & $\begin{array}{c}28.2 \\
(15802)\end{array}$ & $\begin{array}{c}57.2 \\
(7734)\end{array}$ & $\begin{array}{c}39.5 \\
(13413)\end{array}$ \\
\hline$N$ & 903 & 879 & 1008 & 408 & 2689 & 2534 & 2511 & 1142 & 50604 & 60007 & 14343 & 33018 \\
\hline $\begin{array}{l}\text { Proportion of boys aged } 18-20 \\
\text { completed higher-secondary } \\
\text { education }\end{array}$ & $\begin{array}{l}18.0 \\
(157)\end{array}$ & $\begin{array}{l}25.7 \\
(217)\end{array}$ & $\begin{array}{l}36.4 \\
(367)\end{array}$ & $\begin{array}{l}41.0 \\
(161)\end{array}$ & $\begin{array}{l}20.1 \\
(540)\end{array}$ & $\begin{array}{l}28.1 \\
(710)\end{array}$ & $\begin{array}{c}42.9 \\
(1083)\end{array}$ & $\begin{array}{l}42.9 \\
(496)\end{array}$ & $\begin{array}{c}21.2 \\
(9291)\end{array}$ & $\begin{array}{c}24.2 \\
(14404)\end{array}$ & $\begin{array}{c}51.5 \\
(7411)\end{array}$ & $\begin{array}{c}41.6 \\
(13321)\end{array}$ \\
\hline
\end{tabular}

DLHS - District Level Household and Facility Surveys, NFHS - National Family Health Survey.

"9 districts include - Bidar, Gulbarga, Bijapur, Yadgir, Bagalkot, Raichur, Gadag, Koppal and Bellary.

†Data not collected from 8 poorly performing States in India (as data from these States were collected in a different survey). 
Table 6. Profile of rural Scheduled Caste /Scheduled Tribe boys

\begin{tabular}{|c|c|c|c|c|c|c|c|c|c|c|c|c|}
\hline \multirow{4}{*}{ Indicators } & \multicolumn{4}{|c|}{$\begin{array}{l}\text { Northern Karnataka Districts } \\
\text { ( } 9 \text { districts)* }\end{array}$} & \multicolumn{4}{|c|}{ Karnataka } & \multicolumn{4}{|c|}{ India } \\
\hline & $\begin{array}{l}\text { DLHS } \\
\text { II }\end{array}$ & $\begin{array}{l}\text { DLHS } \\
\text { III }\end{array}$ & $\begin{array}{l}\text { DLHS } \\
\text { IV }\end{array}$ & $\begin{array}{l}\text { NFHS } \\
\text { IV }\end{array}$ & $\begin{array}{l}\text { DLHS } \\
\text { II }\end{array}$ & $\begin{array}{l}\text { DLHS } \\
\text { III }\end{array}$ & $\begin{array}{l}\text { DLHS } \\
\text { IV } †\end{array}$ & $\begin{array}{l}\text { NFHS } \\
\text { IV }\end{array}$ & $\begin{array}{l}\text { DLHS } \\
\text { II }\end{array}$ & $\begin{array}{l}\text { DLHS } \\
\text { III }\end{array}$ & $\begin{array}{l}\text { DLHS } \\
\text { IV } †\end{array}$ & $\begin{array}{l}\text { NFHS } \\
\text { IV }\end{array}$ \\
\hline & $2002 / 3$ & $2007 / 8$ & $2012 / 3$ & $2015 / 6$ & $2002 / 3$ & $2007 / 8$ & $2012 / 3$ & $2015 / 6$ & $2002 / 3$ & $2007 / 8$ & $2012 / 3$ & $2015 / 6$ \\
\hline & $\%(N)$ & $\%(N)$ & $\%(N)$ & $\%(N)$ & $\%(N)$ & $\%(N)$ & $\%(\mathrm{~N})$ & $\%(N)$ & $\%(N)$ & $\%(N)$ & $\%(N)$ & $\%(N)$ \\
\hline \multicolumn{13}{|l|}{ Education: } \\
\hline $\mathrm{N}$ & 439 & 554 & 696 & 329 & 1100 & 1174 & 1461 & 748 & 32701 & 43649 & 15129 & 27863 \\
\hline $\begin{array}{l}\text { Proportion of boys aged } 12-14 \\
\text { entered into secondary school }\end{array}$ & $\begin{array}{l}14.3 \\
(60)\end{array}$ & $\begin{array}{r}22.1 \\
(119)\end{array}$ & $\begin{array}{c}20.0 \\
(141)\end{array}$ & $\begin{array}{l}25.2 \\
(79)\end{array}$ & $\begin{array}{l}20.9 \\
(222)\end{array}$ & $\begin{array}{l}23.0 \\
(271)\end{array}$ & $\begin{array}{c}28.2 \\
(412)\end{array}$ & $\begin{array}{l}32.3 \\
(237)\end{array}$ & $\begin{array}{c}21.6 \\
(4824)\end{array}$ & $\begin{array}{c}16.6 \\
(7218)\end{array}$ & $\begin{array}{c}30.6 \\
(4559)\end{array}$ & $\begin{array}{c}24.7 \\
(6318)\end{array}$ \\
\hline$N$ & 359 & 476 & 623 & 275 & 918 & 1080 & 1382 & 666 & 28171 & 39042 & 14693 & 25494 \\
\hline $\begin{array}{l}\text { Proportion of boys aged } 15-17 \\
\text { completed secondary education }\end{array}$ & $\begin{array}{l}16.6 \\
(58)\end{array}$ & $\begin{array}{r}25.2 \\
(122)\end{array}$ & $\begin{array}{r}29.6 \\
(182)\end{array}$ & $\begin{array}{l}37.2 \\
(94)\end{array}$ & $\begin{array}{c}19.3 \\
(175)\end{array}$ & $\begin{array}{l}31.9 \\
(345)\end{array}$ & $\begin{array}{l}37.9 \\
(526)\end{array}$ & $\begin{array}{l}44.4 \\
(291)\end{array}$ & $\begin{array}{c}17.8 \\
(4032)\end{array}$ & $\begin{array}{c}17.2 \\
(6718)\end{array}$ & $\begin{array}{c}39.2 \\
(5706)\end{array}$ & $\begin{array}{c}28.7 \\
(6676)\end{array}$ \\
\hline$N$ & 421 & 526 & 756 & 302 & 1178 & 1270 & 1652 & 716 & 30657 & 43316 & 15410 & 24485 \\
\hline $\begin{array}{l}\text { Proportion of boys aged 18-20 } \\
\text { completed higher-secondary } \\
\text { education }\end{array}$ & $\begin{array}{l}8.8 \\
(39)\end{array}$ & $\begin{array}{l}17.8 \\
(92)\end{array}$ & $\begin{array}{r}24.5 \\
(184)\end{array}$ & $\begin{array}{l}34.6 \\
(98)\end{array}$ & $\begin{array}{l}11.5 \\
(130)\end{array}$ & $\begin{array}{l}20.9 \\
(266)\end{array}$ & $\begin{array}{l}27.7 \\
(459)\end{array}$ & $\begin{array}{c}34.6 \\
(241)\end{array}$ & $\begin{array}{c}14.4 \\
(3624)\end{array}$ & $\begin{array}{c}14.1 \\
(6164)\end{array}$ & $\begin{array}{c}31.9 \\
(4887)\end{array}$ & $\begin{array}{c}28.6 \\
(6136)\end{array}$ \\
\hline
\end{tabular}

DLHS - District Level Household and Facility Surveys, NFHS - National Family Health Survey

"9 districts include - Bidar, Gulbarga, Bijapur, Yadgir, Bagalkot, Raichur, Gadag, Koppal and Bellary.

†Data not collected from 8 poorly performing States in India (as data from these States were collected in a different survey). 


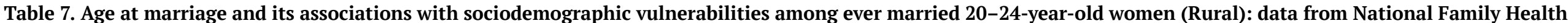
Survey-4

\begin{tabular}{|c|c|c|c|c|c|c|c|c|c|c|c|c|}
\hline & \multicolumn{6}{|c|}{ Karnataka } & \multicolumn{6}{|c|}{ India } \\
\hline & \multicolumn{2}{|c|}{$\begin{array}{c}\text { Scheduled caste/scheduled } \\
\text { tribe }(\mathrm{N}=2078)\end{array}$} & \multicolumn{2}{|c|}{$\begin{array}{l}\text { Poorest quantile } \\
\qquad(\mathrm{N}=2260)\end{array}$} & \multicolumn{2}{|c|}{ Illiterate $(\mathrm{N}=2260)$} & \multicolumn{2}{|c|}{$\begin{array}{c}\text { Scheduled caste/scheduled } \\
\text { tribe }(\mathrm{N}=69454)\end{array}$} & \multicolumn{2}{|c|}{$\begin{array}{l}\text { Poorest quantile } \\
\qquad(\mathrm{N}=72135)\end{array}$} & \multicolumn{2}{|c|}{ Illiterate $(\mathrm{N}=72135)$} \\
\hline & $\mathrm{OR}(95 \% \mathrm{Cl})$ & $P$-value & $\begin{array}{l}\text { OR }(95 \% \\
\mathrm{Cl})\end{array}$ & $P$-value & $\begin{array}{c}\text { OR }(95 \% \\
\text { CI) }\end{array}$ & $P$-value & OR $(95 \% \mathrm{Cl})$ & $P$-value & $\begin{array}{l}\text { OR }(95 \% \\
\mathrm{Cl})\end{array}$ & $P$-value & $\begin{array}{c}\text { OR }(95 \% \\
\mathrm{Cl})\end{array}$ & $P$-value \\
\hline \multicolumn{13}{|c|}{ Age at marriage: } \\
\hline $20-24$ & Ref & & Ref & & Ref & & Ref & & Ref & & Ref & \\
\hline $18-19$ & $\begin{array}{c}1.01 \\
(0.77-1.31)\end{array}$ & 0.969 & $\begin{array}{c}1.37 \\
(0.98-1.91)\end{array}$ & 0.063 & $\begin{array}{c}1.44 \\
(0.98-2.11)\end{array}$ & 0.061 & $\begin{array}{c}1.14 \\
(1.09-1.20)\end{array}$ & 0.000 & $\begin{array}{c}1.77 \\
(1.65-1.89)\end{array}$ & 0.000 & $\begin{array}{c}1.46 \\
(1.37-1.56)\end{array}$ & 0.000 \\
\hline $16-17$ & $\begin{array}{c}1.36 \\
(1.02-1.82)\end{array}$ & 0.037 & $\begin{array}{c}1.60 \\
(1.13-2.27)\end{array}$ & 0.009 & $\begin{array}{c}2.26 \\
(1.52-3.36)\end{array}$ & 0.000 & $\begin{array}{c}1.25 \\
(1.18-1.32)\end{array}$ & 0.000 & $\begin{array}{c}2.38 \\
(2.21-2.55)\end{array}$ & 0.000 & $\begin{array}{c}2.09 \\
(1.95-2.23)\end{array}$ & 0.000 \\
\hline$<16$ & $\begin{array}{c}1.12 \\
(0.79-1.60)\end{array}$ & 0.517 & $\begin{array}{c}2.49 \\
(1.69-3.68)\end{array}$ & 0.000 & $\begin{array}{c}3.81 \\
(2.50-5.82)\end{array}$ & 0.000 & $\begin{array}{c}1.23 \\
(1.15-1.31)\end{array}$ & 0.000 & $\begin{array}{c}2.93 \\
(2.71-3.16)\end{array}$ & 0.000 & $\begin{array}{c}3.39 \\
(3.16-3.64)\end{array}$ & 0.000 \\
\hline
\end{tabular}

$\mathrm{CI}$ - confidence interval, OR - odds ratio 
Table 8. School attainment and its associations with sociodemographic vulnerabilities among girls (Rural): data from National Family Health Survey-4

\begin{tabular}{|c|c|c|c|c|c|c|c|c|c|c|c|c|}
\hline & \multicolumn{6}{|c|}{ Karnataka } & \multicolumn{6}{|c|}{ India } \\
\hline & \multicolumn{2}{|c|}{$\begin{array}{l}\text { Scheduled caste/ } \\
\text { scheduled tribe }\end{array}$} & \multicolumn{2}{|c|}{ Poorest quantile } & \multicolumn{2}{|c|}{$\begin{array}{l}\text { Married/Married but gauna } \\
\text { not performed }\end{array}$} & \multicolumn{2}{|c|}{$\begin{array}{l}\text { Scheduled caste/ } \\
\text { scheduled tribe }\end{array}$} & \multicolumn{2}{|c|}{ Poorest quantile } & \multicolumn{2}{|c|}{$\begin{array}{l}\text { Married/Married but gauna } \\
\text { not performed }\end{array}$} \\
\hline & $\begin{array}{l}\text { OR }(95 \% \\
\text { Cl) }\end{array}$ & $P$-value & $\begin{array}{l}\text { OR }(95 \% \\
\text { CI) }\end{array}$ & $P$-value & OR $(95 \% \mathrm{Cl})$ & $P$-value & $\begin{array}{l}\text { OR }(95 \% \\
\text { CI) }\end{array}$ & $P$-value & $\begin{array}{l}\text { OR }(95 \% \\
\text { CI) }\end{array}$ & $P$-value & OR $(95 \% \mathrm{Cl})$ & $P$-value \\
\hline \multicolumn{13}{|c|}{ Girls aged 12-14 entered into secondary school (Class 8): } \\
\hline $\mathrm{N}$ & 1799 & & 1956 & & 1297 & & 62928 & & 65691 & & 42132 & \\
\hline Yes & Ref & & Ref & & Ref & & Ref & & Ref & & Ref & \\
\hline No & $\begin{array}{c}1.34 \\
(1.06-1.68)\end{array}$ & 0.013 & $\begin{array}{c}1.81 \\
(1.40-2.33)\end{array}$ & 0.000 & $\begin{array}{c}0.47 \\
(0.20-1.08)\end{array}$ & 0.074 & $\begin{array}{c}1.22 \\
(1.16-1.28)\end{array}$ & 0.000 & $\begin{array}{c}2.53 \\
(2.39-2.68)\end{array}$ & 0.000 & $\begin{array}{c}0.94 \\
(0.79-1.12)\end{array}$ & 0.479 \\
\hline \multicolumn{13}{|c|}{ Girls aged 15-17 completed secondary education (Class 10$):$} \\
\hline $\mathrm{N}$ & 1462 & & 1601 & & 1601 & & 57075 & & 59398 & & 59398 & \\
\hline Yes & Ref & & Ref & & Ref & & Ref & & Ref & & Ref & \\
\hline No & $\begin{array}{c}1.69 \\
(1.33-2.14)\end{array}$ & 0.000 & $\begin{array}{c}2.48 \\
(1.88-3.29)\end{array}$ & 0.000 & $\begin{array}{c}1.86 \\
(1.16-2.99)\end{array}$ & 0.010 & $\begin{array}{c}1.52 \\
(1.45-1.59)\end{array}$ & 0.000 & $\begin{array}{c}4.17 \\
(3.90-4.46)\end{array}$ & 0.000 & $\begin{array}{c}2.05 \\
(1.85-2.26)\end{array}$ & 0.000 \\
\hline \multicolumn{13}{|c|}{ Girls aged $18-20$ completed higher-secondary education (Class 12): } \\
\hline $\mathrm{N}$ & 1740 & & 1887 & & 1887 & & 59931 & & 62560 & & 62560 & \\
\hline Yes & Ref & & Ref & & Ref & & Ref & & Ref & & Ref & \\
\hline No & $\begin{array}{c}1.96 \\
(1.54-2.48)\end{array}$ & 0.000 & $\begin{array}{c}3.65 \\
(2.61-5.12)\end{array}$ & 0.000 & $\begin{array}{c}5.56 \\
(4.29-7.22)\end{array}$ & 0.000 & $\begin{array}{c}1.81 \\
(1.72-1.90)\end{array}$ & 0.000 & $\begin{array}{c}4.91 \\
(4.53-5.33)\end{array}$ & 0.000 & $\begin{array}{c}4.37 \\
(4.16-4.60)\end{array}$ & 0.000 \\
\hline
\end{tabular}

CI - confidence interval, OR - odds ratio 
tions, including: 4.1 (ensuring all girls and boys complete free, equitable and quality primary and secondary education), 4.5 (eliminating gender disparities in education), 5.3 (eliminating child marriage), 3.1 (reducing global maternal mortality) and 3.2 (ending preventable deaths of newborns and children under 5 years). ${ }^{30}$

Key strengths of these data include the representativeness of the sample, the robustness of the data and the measures, and the ability to get a range of information from a single survey. However, by analysing the data by caste and by gender, some cells become small, meaning there is a larger amount of uncertainty around the percentages. In addition, the DLHS-IV survey excluded data from eight of the poorest performing States in India (as data from these states was collected using a different survey); exclusion of these states would have resulted in improved levels for some indicators at this time point. As the questionnaires were administered face-to-face, the variables included in our analysis may have been subject to reporting bias, resulting in, for example, under-reporting of child marriage in the household questionnaire, and over-reporting of school retention. Reporting biases may have increased after the introduction of legislation and awareness-raising campaigns regarding these issues among the general population in 2006 and 2009, respectively. ${ }^{22,23}$ The interviewing of evermarried girls and women for the women's questionnaire necessarily skews our comparison group, meaning we could only compare pregnancy and literacy outcomes among married women aged 19-24 years and not among all women aged 19-24 years. This may have led to underestimates of the impact of child marriage on these outcomes. Ecological analysis of cross-sectional data precludes the ability to make causal inferences.

\section{CONCLUSIONS}

Taken together, our analyses suggest that the lack of impact seen in the Samata trial may have been a result of the large secular changes occurring in India during this period. Future trials should assess background trends before investing in a community randomized assessment of project impacts. Unlike the earlier Millennium Development Goals (MDGs), the SDGs include a commitment to "Leave No one Behind," even those who by virtue of intersecting inequalities and stigma are hardest to reach. ${ }^{31}$ We recommend that to benefit this group, future efforts should focus explicitly on the most disadvantaged families and start $1-2$ years before young girls reach menarche. ${ }^{32-35}$ More broadly to achieve the SDGs, India must shift its focus now from enrolment, to improving the quality of education. ${ }^{12}$

\section{ACKNOWLEDGEMENTS}

We would like to thank our colleagues Dr Satyanarayana Ramanaik, Dr Shajy Isac, Ms Martine Collumbien, Ms Parinita Bhattacharjee, Mr Raghavendra Thalinja and Prof Charlotte Watts from the Samata research team, for their intellectual input on the issues of girl child marriage and secondary school drop-out over the past 7 years.

\section{ETHICS APPROVAL}

As this analysis used data from pre-existing nationally-representative datasets from India, no additional ethical approval was sought for the analyses presented in this manuscript.

\section{FUNDING}

This research was funded by UK Aid from the Department for International Development (DFID) as part of the STRIVE Research Programme Consortium, a 7-year programme of research and action devoted to tackling the structural drivers of HIV (http://STRIVE.lshtm.ac.uk/). The views expressed herein are those of the authors and do not necessarily reflect the official policy or position of the UK government. The funding source did not play a role in the design of the study, data analysis, interpretation, or writing of the results. The authors had full access to all the data in the study and had final responsibility for the decision to submit for publication.

\section{COMPETING INTERESTS}

The authors have completed the Unified Competing Interest form at http://www.icmje.org/coi_disclosure.pdf (available on request from the corresponding author) and declare no conflicts of interest.

\section{CORRESPONDENCE TO}

\section{Dr Tara S Beattie}

London School of Hygiene \& Tropical Medicine

15-17 Tavistock Place

London WC1H 9SH, UK

Tara.Beattie@lshtm.ac.uk 


\section{REFERENCES}

1. Raj A, Boehmer U. Girl child marriage and its association with national rates of HIV, maternal health, and infant mortality across 97 countries. Violence Against Women. 2013;19(4):536-551. doi:10.1 $\underline{177 / 1077801213487747}$

2. Wodon Q, Male C, Nayihouba A, Onagoruwa A, Savadogo A, Yedan A, et al. Economic Impacts of Child Marriage: Global Synthesis Report. The World Bank and International Centre for Research on Women; 2017.

3. Kidman R. Child marriage and intimate partner violence: a comparative study of 34 countries. Int J Epidemiol. 2017;46:662-675.

4. Blum RW, Gates W Sr. Girlhood Not Motherhood. Preventing Adolescent Pregnancy. United Nations Population Fund (UNFPA); 2015.

5. Patra S. Motherhood in childhood: addressing reproductive health hazards among adolescent married women in India. Reprod Health. 2016;13(1):52. doi:10.1186/s12978-016-0171-7

6. Raj A. When the mother is a child: the impact of child marriage on the health and human rights of girls. Arch Dis Child. 2010;95(11):931-935. doi:10.113 6/adc. 2009.178707

7. Nguyen MC, Wodon Q. Global Trends in Child Marriage.; 2012. Accessed January 17, 2019. http://ww w.ungei.org/files/Child Marriage Trends3.pdf

8. UNICEF. Ending Child Marriage: Progress and Prospects. UNICEF; 2014.

9. Ramanaik S, Collumbien M, Prakash R, et al. Education, poverty and "purity" in the context of adolescent girls' secondary school retention and dropout: A qualitative study from Karnataka, southern India. PLoS ONE. 2018;13(9):e0202470. do i:10.1371/journal.pone.0202470

10. Roest J. Child Marriage and Early Child-Bearing in India: Risk Factors and Policy Implications. Young Lives; 2016.

11. Sheehan P, Sweeny K, Rasmussen B, et al. Building the foundations for sustainable development: a case for global investment in the capabilities of adolescents. Lancet. 2017;390(10104):1792-1806. do $\mathrm{i}: 10.1016 / \mathrm{s} 0140-6736(17) 30872-3$
12. UNESCO. Education for All 2000-2015: Achievements and Challenges. UNESCO; 2015. Accessed January 17, 2019. https://en.unesco.org/ge m-report/report/2015/education-all-2000-2015-achie vements-and-challenges

13. United Nations. The Millennium Development Goals Report. UN; 2013. Accessed January 17, 2019. http://w ww.un.org/millenniumgoals/pdf/report-2013/mdg-re port-2013-english.pdf

14. Viner RM, Ozer EM, Denny S, et al. Adolescence and the social determinants of health. Lancet. 2012;379(9826):1641-1652. doi:10.1016/s0140-6736(1 2)60149-4

15. Patton GC, Sawyer SM, Santelli JS, et al. Our future: a Lancet commission on adolescent health and wellbeing. Lancet. 2016;387(10036):2423-2478. d oi:10.1016/s0140-6736(16)00579-1

16. Gakidou E, Cowling K, Lozano R, Murray CJ. Increased educational attainment and its effect on child mortality in 175 countries between 1970 and 2009: a systematic analysis. Lancet. 2010;376(9745):959-974. doi:10.1016/s0140-6736(1 0)61257-3

17. Stoner MCD, Pettifor A, Edwards JK, et al. The effect of school attendance and school dropout on incident HIV and HSV-2 among young women in rural South Africa enrolled in HPTN 068. AIDS. 2017;31(15):2127-2134. doi:10.1097/qad.0000000000 $\underline{001584}$

18. Hunt F. Dropping Out from School: A Cross Country Review of the Literature. University of Sussex Centre for International Education; 2008.

19. Roby JL, Erickson L, Nagaishi C. Education for children in sub-Saharan Africa: Predictors impacting school attendance. Child Youth Serv Rev. 2016;64:110-116. doi:10.1016/j.childyouth.2016.03.0 $\underline{02}$

20. Bhagavatheeswaran L, Nair S, Stone H, et al. The barriers and enablers to education among scheduled caste and scheduled tribe adolescent girls in northern Karnataka, South India: A qualitative study. Int J Educ Dev. 2016;49:262-270. doi:10.1016/j.ijedudev.2016.0 $\underline{4.004}$

21. Warrington M, Kiragu S. "It makes more sense to educate a boy”: Girls 'against the odds' in Kajiado, Kenya. Int J Educ Dev. 2012;32(2):301-309. doi:10.101 6/j.ijedudev.2011.05.004 
22. Prohibition of Child Marriage Act.; 2006. Accessed January 17, 2019. http://ncw.nic.in/acts/pcma2006.pd f

23. The Right of Children to Free and Compulsory Education Act.; 2009. Accessed January 17, 2019. http s://indiacode.nic.in/bitstream/123456789/2086/1/A20 09-35.pdf

24. Pillai P, Srikantamurthy HS, Raghavendra T, Vadde K, Hiremath T. Know Your Schemes: An Information Manual on Government Programmes for School-Going Girls. Karnataka Health Promotion Trust; 2014. http://strive.lshtm.ac.uk/sites/strive.lsht m.ac.uk/files/Know\%20your\%20schemes\%20-\%20A n\%20information\%20manual\%20on\%20governmen t\%20programmes\%20for\%20school\%20going\%20girl s.pdf

25. Beattie TS, Bhattacharjee $P$, Isac $S$, et al. Supporting adolescent girls to stay in school, reduce child marriage and reduce entry into sex work as HIV risk prevention in north Karnataka, India: protocol for a cluster randomised controlled trial. BMC Public Health. 2015;15(1):292. doi:10.1186/s12889-015-162 $\underline{3-7}$

26. Prakash R, Beattie T, Javalkar P, Bhattacharjee $P$, Ramanaik S, Thalinja R. The Samata intervention to increase secondary school completion and reduce child marriage among adolescent girls: results from a cluster-randomised control trial in India. J Glob Health. 2019;1(010430). doi:10.7189/jogh.09.010430

27. International Institute for Population Sciences, ICF. India National Family Health Survey (NFHS-4) 2015-16. IIPS; 2017. http://rchiips.org/nfhs/NFHS-4R eports/India.pdf

28. Government of Karnataka. Karnataka Human Development Report. Government of Karnataka; 2006. http://planningcommission.nic.in/plans/stateplan/sd r pdf/shdr kar05.pdf
29. UNESCO Institute for Statistics, Global Education Monitoring Report. Leaving No One Behind: How Far on the Way to Universal Primary and Secondary Education? UIS and GEMR; 2016. https://unesdoc.une sco.org/ark:/48223/pf0000245238_eng

30. United Nations. Sustainable Develoment Goals. UN; 2015. Accessed January 17, 2019. https://www.un.org/ sustainabledevelopment/sustainable-development-go $\underline{\text { als }}$

31. Women UN. Turning Promises into Action: Gender Equality in the 2030 Agenda for Sustainable Development.; 2018.

32. Bruce J. Commentary: Investing in the poorest girls in the poorest communities early enough to make a difference. Glob Public Health. 2015;10(2):225-227. doi:10.1080/17441692.2014.9861 $\underline{70}$

33. Amin S, Ahmed J, Saha J, Hossain Md, Haque E. Delaying Child Marriage through Community-Based Skills-Development Programs for Girls. Results from a Randomized Controlled Study in Rural Bangladesh. Population Council; 2016. https://doi.org/10.31899/p gy9.1009

34. Field E, Glennerster R, Nazneen S, Pimkina S, Sen I, Buchmann N. Age at Marriage, Women's Education, and Mother and Child Outcomes in Bangladesh. In: Impact Evaluation Report 68. International Initiative for Impact Evaluation; 2018. Accessed January 17, 2019. https://www.3ieimpact.org/sites/default/files/2 018-08/IE\%2068-Bangladesh-marriage.pdf

35. Jensen R. Do labor market opportunities affect young women's work and family decisions? Experimental evidence from India. $Q J$ Econ. 2012;127(2):753-792. doi:10.1093/qje/qjs002 